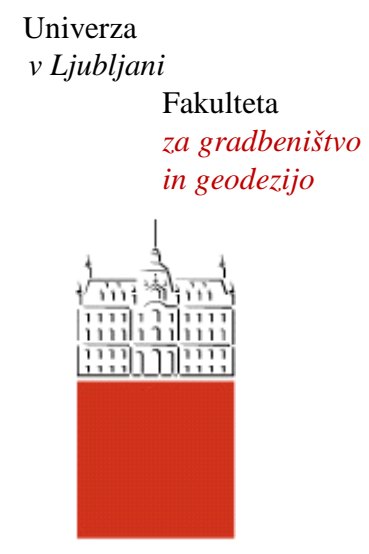

Jamova 2

1000 Ljubljana, Slovenija http://www3.fgg.uni-lj.si/

DRUGG - Digitalni repozitorij UL FGG http://drugg.fgg.uni-lj.si/

Ta članek je avtorjeva zadnja recenzirana različica, kot je bila sprejeta po opravljeni recenziji.

Prosimo, da se pri navajanju sklicujte na bibliografske podatke, kot je navedeno:
University
of Ljubljana

Faculty of

Civil and Geodetic

Engineering

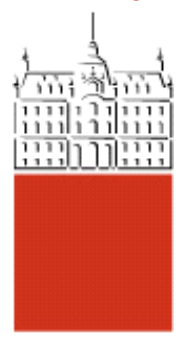

Jamova 2

SI - 1000 Ljubljana, Slovenia

http://www3.fgg.uni-lj.si/

DRUGG - The Digital Repository http://drugg.fgg.uni-lj.si/

This version of the article is author's manuscript as accepted for publishing after the review process.

When citing, please refer to the publisher's bibliographic information as follows:

Brank, B., Perić, D., Damjanić, F. 1997. On large deformations of thin elasto-plastic shells: implementation of a finite rotation model for quadrilateral shell element. Int. j. numer. methods eng., 40, 4: 689-726.

http://onlinelibrary.wiley.com/doi/10.1002/(SICI)1097-0207(19970228)40:4\%3C689::AIDNME85\%3E3.0.CO;2-7/abstract 


\title{
ON LARGE DEFORMATIONS OF THIN ELASTO-PLASTIC SHELLS: IMPLEMENTATION OF A FINITE ROTATION MODEL FOR QUADRILATERAL SHELL ELEMENT
}

\author{
BOŠTJAN BRANK \\ Institute of Structural and Earthquake Engineering, University of Ljubljana, Jamova 2, 61000 Ljubljana, Slovenia \\ DJORDJE PERIĆ \\ Department of Civil Engineering, University of Wales, Swansea, Swansea SA2 8PP, U.K. \\ FRANO B. DAMJANIĆ \\ Institute of Structural and Earthquake Engineering, University of Ljubljana, Jamova 2, 61000 Ljubljana, Slovenia
}

\begin{abstract}
SUMMARY
A large-deformation model for thin shells composed of elasto-plastic material is presented in this work. Formulation of the shell model, equivalent to the two-dimensional Cosserat continuum, is developed from the three-dimensional continuum by employing standard assumptions on the distribution of the displace- ment field in the shell body. A model for thin shells is obtained by an approximation of terms describing the shell geometry. Finite rotations of the director field are described by a rotation vector formulation. An elasto-plastic constitutive model is developed based on the von Mises yield criterion and isotropic hardening. In this work, attention is restricted to problems where strains remain small allowing for all aspects of material identification and associated computational treatment, developed for small-strain elasto- plastic models, to be transferred easily to the present elasto-plastic thin-shell model. A finite element formulation is based on the four-noded isoparametric element. A particular attention is devoted to the consistent linearization of the shell kinematics and elasto-plastic material model, in order to achieve quadratic rate of asymptotic convergence typical for the Newton-Raphson-based solution procedures. To illustrate the main objective of the present approach — namely the simulation of failures of thin elasto- plastic shells typically associated with buckling-type instabilities and/or bending-dominated shell problems resulting in formation of plastic hinges - several numerical examples are presented. Numerical results are compared with the available experimental results and representative numerical simulations.
\end{abstract}

KEY WORDS: shells; large deformations; finite rotations; elasto-plasticity; finite elements

\section{INTRODUCTION}

This work is concerned with formulation and computational aspects of the elasto-plastic model for thin shells. A simple von Mises yield criterion with isotropic hardening is adopted in constitutive description of elasto-plastic material. Attention is restricted to situations where strains remain infinitesimal, although continuum rotations are not restricted. Details and 
motivation for the present constitutive description may be found in Reference 1 while numerical treatment follows References 2 and 3.

In problems where finite rotations may be present but strains are restricted to be small, the adopted von Mises model for large deformations of elasto-plastic shells is equivalent to the small-strain von Mises elasto-plastic model. Accordingly, all aspects of material identification and associated computational procedures known for small-strain elasto-plastic models transfer easily to the present elasto-plastic thin-shell model. For situations arising during failures of elasto-plastic thin shells typically associated with buckling-type instabilities and/or bendingdominated shell problems resulting in the formation of plastic hinges, this model is expected to provide accurate description of the physical phenomena.

Modern finite element formulations incorporating non-linear shell kinematics arise from the shell theories, which are based either on the direct approach ${ }^{4}$ or derived from the three-

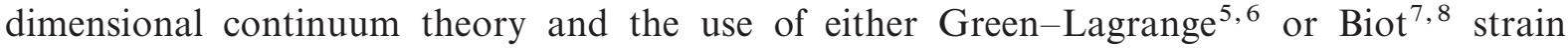
measures. The strain measures are typically restricted to be small.

A strong analogy of the formulations based on the degenerated continuum approach (see e.g. Reference 6) and those based on shell theories can be noticed (see Reference 9), if a certain version of an explicit integration or a numerical integration through the shell thickness are performed in the former. For instance, Büchter and Ramm ${ }^{9}$ showed that the numerical integration across the thickness in the degenerated continuum model leads to the strain tensor, which is consistent with the geometrically non-linear shell theory for small strains including transverse shear deformations. ${ }^{10}$

Finite element formulation described in this work relies on the four-node shell element based on isoparametric interpolation scheme that has been commonly used for the finite element implementation of different shell theories of the Mindlin-Reissner type. In the recent works of Parisch, ${ }^{5}$ Sansour and Bufler, ${ }^{7}$ Simo et al. ${ }^{4,11,12}$ and Wriggers and Gruttmann ${ }^{8}$ the four-node elements take part in non-linear formulations capable of describing large displacements and finite rotations of shells. In this work, the formulation described in Reference 13 is extended to account for large displacements and finite rotations of thin elasto-plastic shells. Large rotations of the director field are described by a rotation vector formulation, while the formulation in Reference 13 is based on two Euler angles.

The displacement-based formulations suffer from the so-called transverse shear locking, a phenomena which is closely connected with the underlying assumptions typical for the shell theories of the Mindlin-Reissner type. It is therefore essential that the solutions are based on mixed variational formulations. A current 'standard' to avoid transverse shear locking is the so-called Assumed Natural Strain (ANS) approach, first suggested by Dvorkin and Bathe. ${ }^{14}$ An important computational aspect of the isoparametric shell elements is also a treatment of membrane locking - a phenomena which is associated with a parasitic membrane strains under pure bending conditions. Since elements with bilinear interpolation do not show a particular sensitivity to this defect, a displacement formulation is used for the membrane and bending parts of a variational formulation.

Local Cartesian frames are defined at numerical integration points in order to simplify the expressions. A computational procedure is provided to include the through-thickness variable material characteristics typically encountered in evolving properties of elasto-plastic materials. This is considered important if accurate simulation of elasto-plastic shells is required.

From the computational point of view, a robust and efficient algorithm with high rate of convergence is required. Consistent linearization of the shell kinematics and elasto-plastic material model leading to the Newton-Raphson numerical procedure is therefore performed. Robustness and efficiency of the proposed approach are illustrated on a range of numerical 
examples. A comparison is made with available experimental results and a representative set of numerical examples.

\section{SHELL KINEMATICS}

\subsection{Preliminaries}

Let a bounded open set $\mathscr{B} \subset \mathbb{R}^{3}$ with a regular boundary $\partial \mathscr{B}$ define a reference placement of the three-dimensional shell-like body. A configuration of a body is a mapping $\chi: \mathscr{B} \rightarrow \mathbb{R}^{3}$. A collection of all configurations of a body forms the so-called configuration space $\mathscr{C}(\mathscr{B})$. A motion is a curve in $\mathscr{C}$. For fixed $t \in[0, T] \subset \mathbb{R}^{+}$mapping $t \mapsto \chi(\bullet, t)$ defines the configuration. We often use the notation $\chi_{t}(\mathbf{X})=\chi(\mathbf{X}, t)$. Points $X$ will be identified with its co-ordinate $\mathbf{X}$ and $\mathbf{x}$ in the reference $\mathscr{B}$ and spatial configuration $\mathscr{S} \ni \chi_{t}(\mathscr{B})$, respectively. Thus $\mathscr{B} \ni \mathbf{X} \rightarrow \mathbf{X}=\chi_{t}(\mathbf{X})$.

Shell is a special type of the three-dimensional body whose one dimension (referred to as thickness) is small compared to some characteristic length of the body (for precise meaning of these terms we refer to Reference 10). In the present work the single director approach is adopted in which the shell-like body is described in terms of a shell (reference) surface $\mathscr{M} \subset \mathscr{B} \subset \mathbb{R}^{3}$ and an independent director field in the thickness direction of the shell body. The director field is not constrained to be normal to the middle surface. Let $\mathscr{A}$ be a bounded open set in $\mathbb{R}^{2}$ with a regular boundary $\partial \mathscr{A}$. Mid-surface then may be parameterized by employing a set of convected coordinates $\left(\xi^{1}, \xi^{2}\right) \in \mathscr{A}$. Mappings $\varphi_{0}\left(\xi^{1}, \xi^{2}\right)$ and $\boldsymbol{\varphi}_{t}\left(\xi^{1}, \xi^{2}\right)$ define the middle surface of the shell at its reference and current configuration, respectively. For each point $\left(\xi^{1}, \xi^{2}\right) \in \mathscr{A}$ unit vectors $\mathbf{T}\left(\xi^{1}, \xi^{2}\right)$ and $\mathbf{t}\left(\xi^{1}, \xi^{2}\right)$ at $\overline{\mathbf{X}}:=\boldsymbol{\varphi}_{0}\left(\xi^{1}, \xi^{2}\right)$ and $\overline{\mathbf{x}}:=\boldsymbol{\varphi}_{t}\left(\xi^{1}, \xi^{2}\right)$ define the corresponding directors. If $h_{0}$ denotes the shell thickness in the reference configuration, the reference configuration of the shell body may be represented as

$$
\mathscr{B}:=\left\{\mathbf{X}=\boldsymbol{\varphi}_{0}\left(\xi^{1}, \xi^{2}\right)+\xi \mathbf{T}\left(\xi^{1}, \xi^{2}\right) \mid\left(\xi^{1}, \xi^{2}\right) \in \mathscr{A}, \xi \in\left[h_{0}^{-}, h_{0}^{+}\right]\right\}
$$

Analogously, the spatial configuration of the shell may be written as

$$
\mathscr{S}:=\left\{\mathbf{x}=\boldsymbol{\varphi}_{t}\left(\xi^{1}, \xi^{2}\right)+\xi \mathbf{t}\left(\xi^{1}, \xi^{2}\right) \mid\left(\xi^{1}, \xi^{2}\right) \in \mathscr{A}, \xi \in\left[h_{0}^{-}, h_{0}^{+}\right]\right\}
$$

In what follows it is convenient to employ the compact notation $\boldsymbol{\Phi}_{0}\left(\mathscr{A} \times\left[h_{0}^{-}, h_{0}^{+}\right]\right)=\mathscr{B}$ and $\boldsymbol{\Phi}_{t}\left(\mathscr{A} \times\left[h_{0}^{-}, h_{0}^{+}\right]\right)=\mathscr{S}$. Clearly,

$$
\chi_{t}=\boldsymbol{\Phi}_{t} \circ \boldsymbol{\Phi}_{0}^{-1}
$$

Illustration of these concepts is provided in Figure 1.

Remark 1. A standard (fixed) basis in $\mathbb{R}^{3}$ is denoted $\left\{\mathbf{e}_{i}\right\}_{i=1,2,3}$. In the following, the vectors $\mathbf{X}$, $\mathbf{T}, \mathbf{x}$ and $\mathbf{t}$ will often be resolved in that basis:

$$
\begin{array}{ll}
\mathbf{X}=X^{i} \mathbf{e}_{i}, & \mathbf{x}=x^{i} \mathbf{e}_{i} \\
\mathbf{T}=T^{i} \mathbf{e}_{i}, & \mathbf{t}=t^{i} \mathbf{e}_{i}
\end{array}
$$

This is a practice which makes shell theories well suited for the numerical treatment. The displacement at a point $\mathbf{X}\left(\xi^{1}, \xi^{2}, \xi\right)$ then may be introduced as (see Figure 2)

$$
\mathbf{U}\left(\xi^{1}, \xi^{2}, \xi\right)=\mathbf{u}\left(\xi^{1}, \xi^{2}\right)+\xi\left(\mathbf{t}\left(\xi^{1}, \xi^{2}\right)-\mathbf{T}\left(\xi^{1}, \xi^{2}\right)\right)
$$




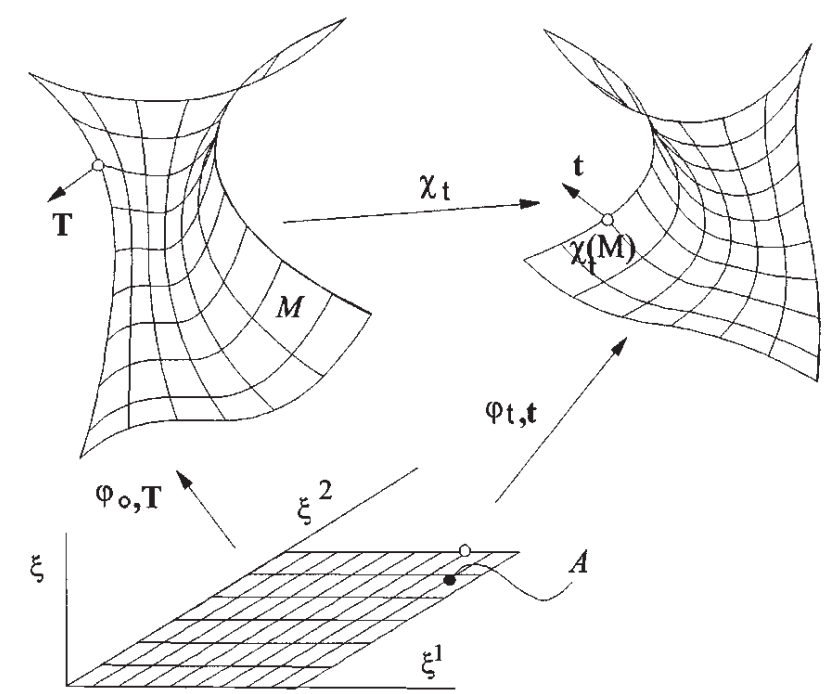

Figure 1. Geometric description of the shell middle surface in reference and current configuration

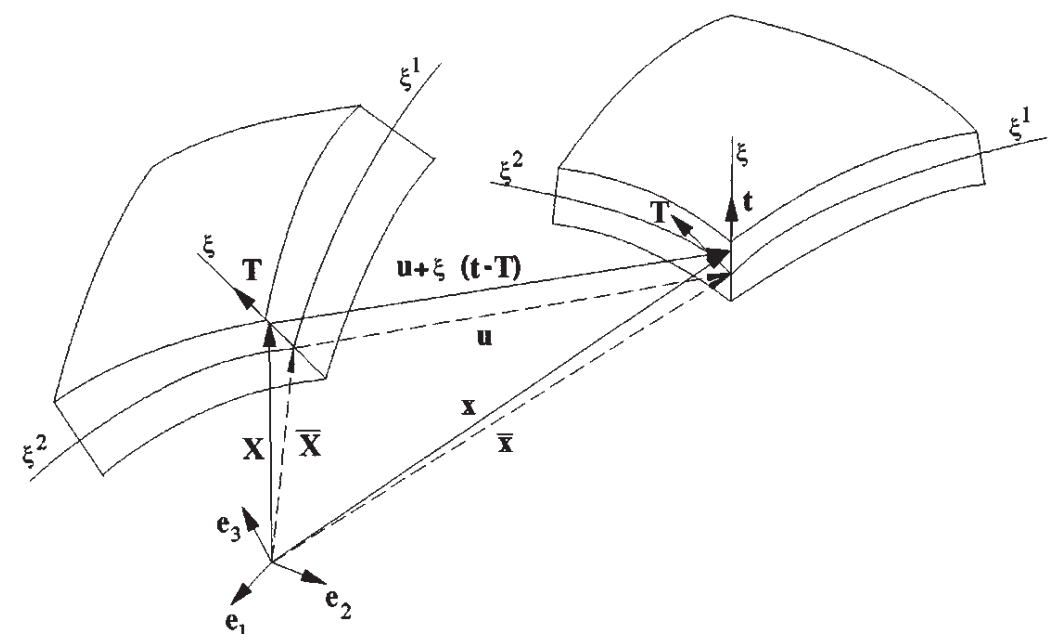

Figure 2. Basic kinematic variables of the shell in reference and current configuration

where

$$
\mathbf{u}\left(\xi^{1}, \xi^{2}\right)=\overline{\mathbf{x}}\left(\xi^{1}, \xi^{2}\right)-\overline{\mathbf{X}}\left(\xi^{1}, \xi^{2}\right)
$$

is the displacement of the middle surface point $\overline{\mathbf{X}}=\boldsymbol{\varphi}_{0}\left(\xi^{1}, \xi^{2}\right)$. It can be observed that the displacement field $\mathbf{U}$ varies linearly over the shell thickness.

\subsection{The deformation gradient}

Let $T_{\mathbf{X}} \mathscr{B} \subset \mathbb{R}^{3}$ and $T_{\mathbf{x}} \mathscr{S} \subset \mathbb{R}^{3}$ denote the tangent spaces to $\mathscr{B}$ and $\mathscr{S}$ at the points $\mathbf{X} \in \mathscr{B}$ and $\mathbf{x} \in \mathscr{S}$. In a standard manner linearly independent vectors

$$
\mathbf{G}_{i}=\frac{\partial \mathbf{X}}{\partial \xi^{i}}, \quad \mathbf{G}_{i} \in T_{\mathbf{X}} \mathscr{B} \quad \text { and } \quad \mathbf{g}_{i}=\frac{\partial \mathbf{x}}{\partial \xi^{i}}, \quad \mathbf{g}_{i} \in T_{\mathbf{x}} \mathscr{S}, \quad \text { for } i=1,2,3
$$


define a basis for the tangent spaces $T \mathscr{B}$ and $T \mathscr{S}$. Deformation gradient is defined as a tangent of the map $\chi_{t}$

$$
T \chi_{t}: T \mathscr{B} \rightarrow T \mathscr{S}, \quad T \chi_{t}=\mathbf{F}: \mathbf{G}_{i} \rightarrow \mathbf{g}_{i}
$$

and is given by a tensor product

$$
\mathbf{F}=\mathbf{g}_{i} \otimes \mathbf{G}^{i}
$$

The contravariant base vector $\mathbf{G}^{i}$ is defined by a standard relation $\mathbf{G}_{j} \cdot \mathbf{G}^{i}=\delta_{j}^{i}\left(\delta_{j}^{i}\right.$ is a Kronecker delta symbol). The basic shell kinematic assumption defines the tangent vectors (6) to be

$$
\begin{gathered}
\mathbf{G}_{\alpha}=\frac{\partial \overline{\mathbf{X}}}{\partial \xi^{\alpha}}+\xi \frac{\partial \mathbf{T}}{\partial \xi^{\alpha}}=\mathbf{A}_{\alpha}+\xi \mathbf{T}_{, \alpha}, \quad \mathbf{g}_{\alpha}=\frac{\partial \overline{\mathbf{x}}}{\partial \xi^{\alpha}}+\xi \frac{\partial \mathbf{t}}{\partial \xi^{\alpha}}=\mathbf{a}_{\alpha}+\xi \mathbf{t}_{, \alpha}, \quad(\alpha=1,2) \\
\mathbf{G}_{3}=\mathbf{a}_{3}=\mathbf{T}, \quad \mathbf{g}_{3}=\mathbf{a}_{3}=\mathbf{t}
\end{gathered}
$$

Vectors $\mathbf{A}_{\alpha}=\left.\mathbf{G}_{\alpha}\right|_{\xi=0}$ define a basis for the tangent space to the middle surface at the point $\overline{\mathbf{X}}\left(\xi^{1}, \xi^{2}\right) \in \mathscr{M}$ and $\mathbf{a}_{\alpha}=\left.\mathbf{g}_{\alpha}\right|_{\xi=0}$ are their images at the actual configuration at the point $\overline{\mathbf{x}}\left(\xi^{1}, \xi^{2}\right) \in \chi_{t}(\mathscr{M})$. The covariant components of the shell body and middle surface metric tensors at the reference configuration are defined by $G_{i j}=\mathbf{G}_{i} \cdot \mathbf{G}_{j}$ and $A_{\alpha \beta}=\mathbf{A}_{\alpha} \cdot \mathbf{A}_{\beta}$, respectively. Their images at the actual configuration are defined by $g_{i j}=\mathbf{g}_{i} \cdot \mathbf{g}_{j}$ and $a_{\alpha \beta}=\mathbf{a}_{\alpha} \cdot \mathbf{a}_{\beta}$. Similarly, their contravariant parts are denoted as $G^{i j}=\mathbf{G}^{i} \cdot \mathbf{G}^{j}, A^{\alpha \beta}=\mathbf{A}^{\alpha} \cdot \mathbf{A}^{\beta}, g^{i j}=\mathbf{g}^{i} \cdot \mathbf{g}^{j}$ and $a^{\alpha \beta}=\mathbf{a}^{\alpha} \cdot \mathbf{a}^{\beta}$. In the present work the director is always chosen to the orthogonal to the middle surface in the reference configuration. Therefore some components of the metric tensor become zero, i.e. $\mathbf{A}_{\alpha} \cdot \mathbf{T}=0, \mathbf{A}^{\alpha} \cdot \mathbf{T}=0$, while, in general, $\mathbf{a}_{\alpha} \cdot \mathbf{t} \neq 0, \mathbf{a}^{\alpha} \cdot \mathbf{t} \neq 0$.

At the reference configuration a mapping $\mathbf{Z}$ can be determined, $\mathbf{Z}: \mathbf{A}_{i} \rightarrow \mathbf{G}_{i}$, which relates metric of the shell body with metric of the middle surface. It may be expressed in the form

$$
\mathbf{Z}=\mathbf{G}_{i} \otimes \mathbf{A}^{i}=\left(A_{\alpha \beta}-\xi B_{\alpha \beta}\right) \mathbf{A}^{\alpha} \otimes \mathbf{A}^{\beta}+\mathbf{T} \otimes \mathbf{T}
$$

where $A_{\alpha \beta} \mathbf{A}^{\alpha} \otimes \mathbf{A}^{\beta}$ is a symmetric tensor of the first fundamental form and $B_{\alpha \beta} \mathbf{A}^{\alpha} \otimes \mathbf{A}^{\beta}$ is a symmetric tensor of the second fundamental form of the reference surface. The components $B_{\alpha \beta}$ are defined as

$$
B_{\alpha \beta}=-\mathbf{A}_{\alpha} \cdot \mathbf{T}_{, \beta}
$$

From the definition (8) and equation (9) deformation gradient $\mathbf{F}$ is obtained as

$$
\mathbf{F}=\left(\mathbf{a}_{\alpha} \otimes \mathbf{G}^{\alpha}+\mathbf{t} \otimes \mathbf{T}\right)+\xi \mathbf{t}_{, \alpha} \otimes \mathbf{G}^{\alpha}
$$

By employing inverse of the tensor $\mathbf{Z}, \mathbf{Z}^{-1}: \mathbf{G}_{i} \rightarrow \mathbf{A}_{i}$ and the relation $\mathbf{G}^{i}=\mathbf{Z}^{-\mathrm{T}} \mathbf{A}^{i}$, the deformation gradient may be expressed as

$$
\mathbf{F}=\left[\left(\mathbf{a}_{\alpha} \otimes \mathbf{A}^{\alpha}+\mathbf{t} \otimes \mathbf{T}\right)+\xi \mathbf{t}_{, \alpha} \otimes \mathbf{A}^{\alpha}\right] \mathbf{Z}^{-1}
$$

It can be observed, from (12) and (13), that the part of deformation gradient related to the middle surface, can be additively split into constant and linear parts.

\subsection{The rotation tensor}

In this section, basic properties of the rotation tensor are described. For further details we refer to References 4, 7, 9, 11, 15 and 16 references therein. 
2.3.1. Basic relations. Any rotation tensor $\mathbf{R} \in \mathbf{S O}(3),\left(\mathbf{R}^{-1}=\mathbf{R}^{\mathrm{T}}\right.$ and $\left.\operatorname{det}[\mathbf{R}]=1\right)$ can be associated with the skew-symmetric tensor $\mathbf{S} \in \operatorname{so}(3)\left(\mathbf{S}=-\mathbf{S}^{\mathbf{T}}\right)$ by the exponential mapping

$$
\mathbf{R}=\exp [\mathbf{S}]=\sum_{k=0}^{\infty} \frac{(\mathbf{S})^{k}}{k !}=\mathbf{1}+\mathbf{S}+\frac{\mathbf{S}^{2}}{2 !}+\frac{\mathbf{S}^{3}}{3 !}+\cdots
$$

Recall that $\mathrm{SO}(3)$ is the group of proper orthogonal transformations (rotations) in $\mathbb{R}^{3}$ (or the Lie group) and so(3) is its associated algebra (or the Lie algebra). Any $\mathbf{S}$ further posseses a so-called axial vector $\mathbf{s} \in \mathbb{R}^{3}$, such that $\mathbf{S s}=0$ and

$$
\mathbf{S b}=\mathbf{s} \times \mathbf{b}, \quad \forall \mathbf{b} \in \mathbb{R}^{3}
$$

Note that the relation (15) defines an isomorphism between so (3) and $\mathbb{R}^{3}$, so (3) $\mapsto \mathbb{R}^{3}: \mathbf{S} \mapsto \mathbf{s}$. By observing that $\mathbf{R s}=\mathbf{s}$, it follows that the axial vector $\mathbf{s}$ is also an eigenvector of $\mathbf{R}$ with eigenvalue 1 ; that is, $\mathbf{R}$ defines rotation about $\mathbf{s}$.

Many possibilities exist for an explicit representation of the exponential mapping. Here we consider a representation in terms of an eigenvector $\mathbf{s}$, also known as the Rodrigues formula,

$$
\mathbf{R}=\cos (\|\mathbf{s}\|) \mathbf{1}+\frac{\sin (\|\mathbf{s}\|)}{\|\mathbf{s}\|} \mathbf{S}+\frac{1-\cos (\|\mathbf{s}\|)}{\|\mathbf{s}\|^{2}} \mathbf{s} \otimes \mathbf{s}
$$

where the norm $\|\mathbf{s}\|$ defines the magnitude of the rotation.

2.3.2. The motion of the director. Motion of the inextensible director may be described as

$$
\mathbf{t}=\mathbf{R T}
$$

where the length of $\mathbf{t}$ is set to be 1 . Clearly, in this case the eigenvector $\mathbf{s}$ is perpendicular to $\mathbf{T}$ and t (see Figure 3)

$$
\mathbf{s} \cdot \mathbf{T}=0, \quad \mathbf{s} \cdot \mathbf{t}=0
$$

and is an element of the tangent space to the middle surface, $\mathbf{s} \in T \mathscr{M}$. By recalling that $(\mathbf{s} \otimes \mathbf{s}) \mathbf{T}=(\mathbf{s} \cdot \mathbf{T}) \mathbf{s}=0$, from (16) it follows that

$$
\mathbf{t}=\mathbf{R} \mathbf{T}=\cos (\|\mathbf{s}\|) \mathbf{T}+\frac{\sin (\|\mathbf{s}\|)}{\|\mathbf{s}\|} \mathbf{s} \times \mathbf{T}
$$

Let the components of $\mathbf{t}, \mathbf{s}$ and $\mathbf{T}$ be defined relative to the standard basis $\left\{\mathbf{e}_{i}\right\}$, so that $\mathbf{s}=s^{i} \mathbf{e}_{i}=s^{1} \mathbf{e}_{1}+s^{2} \mathbf{e}_{2}+s^{3} \mathbf{e}_{3}$ and $\|\mathbf{s}\|=\sqrt{s^{i} s^{i}}$. The components of $\mathbf{t}$ with respect to this basis are

$$
\mathbf{t}=t^{i} \mathbf{e}_{i}=\cos (\|\mathbf{s}\|)\left\{\begin{array}{c}
T^{1} \\
T^{2} \\
T^{3}
\end{array}\right\}+\frac{\sin (\|\mathbf{s}\|)}{\|\mathbf{s}\|}\left\{\begin{array}{c}
-s^{3} T^{2}+s^{2} T^{3} \\
s^{3} T^{1}-s^{1} T^{3} \\
-s^{2} T^{1}+s^{1} T^{2}
\end{array}\right\}
$$

The three components of the vector $\mathbf{s}$, resolved in the fixed basis $\mathbf{e}_{i}$, are not independent because of the constraints (18). It is therefore suitable to choose a different basis, which renders only two independent components. For simplicity, we take such basis to be orthonormal and denote it by $\left\{\mathbf{E}_{i}\right\}_{i=1,2,3}$. It is defined at each point of the middle surface $\mathscr{M}$ with the relations: $\mathbf{E}_{3}=\mathbf{T}$, $\mathbf{E}_{1} \perp \mathbf{E}_{3}, \mathbf{E}_{2}=\mathbf{E}_{3} \times \mathbf{E}_{1}$. Thus $\mathbf{E}_{1}$ and $\mathbf{E}_{2}$ are elements of $T \mathscr{M}$. The components of $\mathbf{s}$, resolved in 


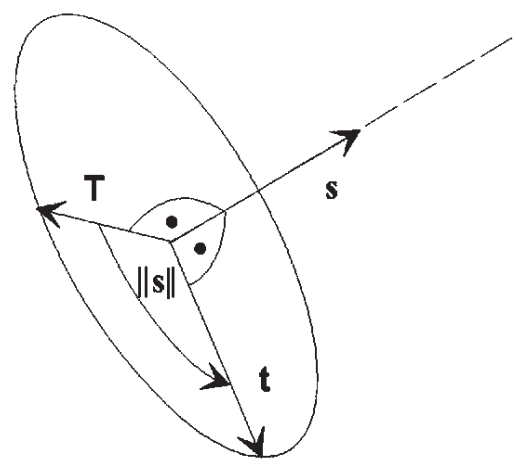

(a)

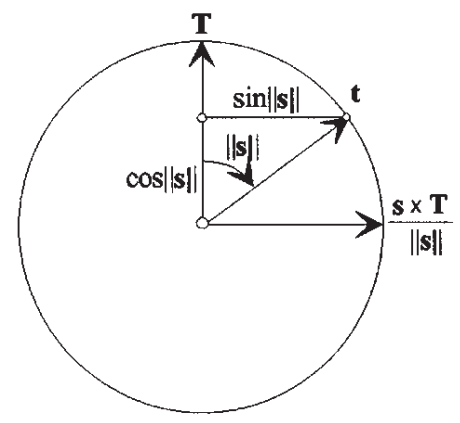

(b)

Figure 3. (a) Rotation of the director. (b) Geometric description of the director rotation

that basis, are $\mathbf{s}=\hat{s}^{1} \mathbf{E}_{1}+\hat{s}^{2} \mathbf{E}_{2}$ while the components of $\mathbf{T}$ are $\mathbf{T}=\hat{T}^{3} \mathbf{E}_{3}=\mathbf{E}_{3}$. The component form of $\mathbf{t}$ with respect to the same basis may be expressed as

$$
\mathbf{t}=\hat{t}^{i} \mathbf{E}_{i}=\cos (\|\mathbf{s}\|)\left\{\begin{array}{l}
0 \\
0 \\
1
\end{array}\right\}+\frac{\sin (\|\mathbf{s}\|)}{\|\mathbf{s}\|}\left\{\begin{array}{c}
\hat{s}^{2} \\
-\hat{s}^{1} \\
0
\end{array}\right\}
$$

The transformation between middle surface basis $\left\{\mathbf{E}_{i}\right\}$ and standard basis $\left\{\mathbf{e}_{i}\right\}$ takes a standard form

$$
\mathbf{E}_{i}=\Lambda^{0} \mathbf{e}_{i}, \quad \text { with } \quad \boldsymbol{\Lambda}^{0}=\left[\mathbf{E}_{1}, \mathbf{E}_{2}, \mathbf{E}_{3}\right], \quad \boldsymbol{\Lambda}^{0} \in \mathrm{SO}(3)
$$

The director, resolved in the fixed basis $\left\{\mathbf{e}_{i}\right\}$, can now be equivalently written in the form

$$
\mathbf{t}=t^{i} \mathbf{e}_{i}=\left[\begin{array}{ccc}
\Lambda_{11}^{0} & \Lambda_{12}^{0} & \Lambda_{13}^{0} \\
\Lambda_{21}^{0} & \Lambda_{22}^{0} & \Lambda_{23}^{0} \\
\Lambda_{31}^{0} & \Lambda_{32}^{0} & \Lambda_{33}^{0}
\end{array}\right]\left\{\cos (\|\mathbf{s}\|)\left\{\begin{array}{l}
0 \\
0 \\
1
\end{array}\right\}+\frac{\sin (\|\mathbf{s}\|)}{\|\mathbf{s}\|}\left\{\begin{array}{c}
\hat{S}^{2} \\
-\hat{s}^{1} \\
0
\end{array}\right\}\right\}
$$

Note, that equations (20) and (23) give the same components of $\mathbf{t}$ in the fixed basis $\left\{\mathbf{e}_{i}\right\}$. In the following, the form (23) will be used, since it leads to two unknown rotational kinematic fields in contrast to the form (20) which gives three rotational kinematic fields with the components being constrained by (18). 


\section{THE CONFIGURATION AND THE TANGENT SPACE}

\subsection{The configuration space}

By employing (17) in (3)-(5) the rotation tensor explicitly appears in the construction of the shell theory and its finite element computational models. Since $\mathbf{T}$ and $\mathbf{t}$ describe the inextensional director field, the collection of all possible configurations, i.e. the configuration space of the shell, may be defined in the following form:

$$
\mathscr{C}=\left\{\boldsymbol{\Phi}=(\boldsymbol{\varphi}, \mathbf{t}) \mid \mathscr{A} \subset \mathbb{R}^{2} \rightarrow \mathbb{R}^{3} \times S^{2}\right\}
$$

where $S^{2}=\left\{\mathbf{t} \in \mathbb{R}^{3} \mid\|\mathbf{t}\|=1\right\}$ denotes a unit sphere, i.e. space of all vectors in $\mathbb{R}^{3}$ satisfying the constraint (18b). The relation between the vector field $\mathbf{t}$ and the primary kinematic variable $\mathbf{s}: \mathscr{A} \rightarrow \mathbb{R}^{3}$, which defines the rotation field, is given by equation (19).

\subsection{The tangent space}

Consider $\mathscr{C}$ as a family of one parameter configurations such that a curve $\boldsymbol{\Phi}:[0, T] \rightarrow \mathscr{C}$ defined by $(\boldsymbol{\varphi}(t), \mathbf{t}(t)) \in \mathscr{C}, t \in[0, T] \subset \mathbb{R}^{+}$, and let further $\left(\boldsymbol{\varphi}\left(t_{o}\right), \mathbf{t}\left(t_{o}\right)\right) \in \mathscr{C}$ describe the configuration at time instant $t=t_{o}$. For sufficiently regular motion, the tangent to the curve $(\varphi(t), \mathbf{t}(t))$ at $t_{o}$ exists and may be defined by

$$
\mathrm{D}(\boldsymbol{\varphi}, \mathbf{t})[\delta \mathbf{u}, \delta \mathbf{t}]=\left.\frac{\mathrm{d}}{\mathrm{d} \varepsilon}\right|_{\varepsilon=0}\left(\boldsymbol{\varphi}_{\varepsilon}, \mathbf{t}_{\varepsilon}\right)=\left.\frac{\mathrm{d}}{\mathrm{d} \varepsilon}\right|_{\varepsilon=0}(\boldsymbol{\varphi}+\varepsilon \delta \mathbf{u}, \mathbf{t}(\mathbf{s}+\varepsilon \delta \mathbf{s}))=:(\delta \mathbf{u}, \delta \mathbf{t})
$$

This, by definition, introduces the space of all admissible variations at the configuration $\left(\boldsymbol{\varphi}\left(t_{o}\right), \mathbf{t}\left(t_{o}\right)\right)$ as a tangent space to $\mathscr{C}$ that is explicitly given as

$$
T_{\Phi} \mathscr{C}=\left\{(\delta \mathbf{u}, \delta \mathbf{t})\left|\delta \mathbf{u}: \mathscr{A} \rightarrow \mathbb{R}^{3}, \delta \mathbf{t}: \mathscr{A} \rightarrow \mathrm{T} S^{2}, \delta \mathbf{u}=0\right|_{\mathscr{M}_{u}}, \delta \mathbf{t}=\left.0\right|_{\mathscr{M}_{t}}\right\}
$$

where $\mathscr{M}_{u}$ and $\mathscr{M}_{t}$ are parts of the middle surface $\mathscr{M}$ where $\mathbf{u}$ and $\mathbf{t}$ are prescribed.

Remark 2. The above-defined configuration and tangent space allow for an additive update of all kinematic variables in the finite element computational process.

\subsection{Variations of kinematic variables}

Linearization of kinematic variables at a given configuration $\boldsymbol{\Phi}=(\boldsymbol{\varphi}, \mathbf{t})$ is performed by a systematic application of the directional derivative to a one-parameter family of configurations described by $\boldsymbol{\Phi}_{\varepsilon}=\left(\boldsymbol{\varphi}_{\varepsilon}, \mathbf{t}_{\varepsilon}\right)$. The variations and/or incremental quantities are then obtained by an ordinary differentiation with respect to $\varepsilon$ as

$$
(\delta \boldsymbol{\varphi}, \delta \mathbf{t}):=\left.\frac{\mathrm{d}}{\mathrm{d} \varepsilon}\right|_{\varepsilon=0}\left(\boldsymbol{\varphi}_{\varepsilon}, \mathbf{t}_{\varepsilon}\right)
$$

Linear structure of $\mathbb{R}^{3}$ provides a simple expression for the curve $\boldsymbol{\varphi}_{\varepsilon}$

$$
\boldsymbol{\varphi}_{\varepsilon}=\boldsymbol{\varphi}+\varepsilon \delta \mathbf{u}
$$

while the one-parameter curve $\mathbf{t}_{\varepsilon}$ is obtained as

$$
\mathbf{S}^{2} \ni \mathbf{t}_{\varepsilon}=\mathbf{R}_{\varepsilon} \mathbf{T}=\mathbf{R}(\mathbf{s}+\varepsilon \delta \mathbf{s}) \mathbf{T}
$$


It then follows, trivially, that

$$
\delta \boldsymbol{\varphi}=\left.\frac{\mathrm{d}}{\mathrm{d} \varepsilon}\right|_{\varepsilon=0} \boldsymbol{\varphi}_{\varepsilon}=\delta \mathbf{u}
$$

By employing (29) and the expression (19) for $\mathbf{t}$ the differentiation of the one-parameter curve $\mathbf{t}_{\varepsilon}$ results in

$$
\delta \mathbf{t}=\frac{\sin (\|\mathbf{s}\|)}{\|\mathbf{s}\|}[-(\mathbf{s} \cdot \delta \mathbf{s}) \mathbf{T}+\delta \mathbf{s} \times \mathbf{T}]+\frac{\|\mathbf{s}\| \cos (\|\mathbf{s}\|)-\sin (\|\mathbf{s}\|)}{\|\mathbf{s}\|^{3}}(\mathbf{s} \cdot \delta \mathbf{s})(\mathbf{s} \times \mathbf{T})
$$

Recalling the connections (see Reference 17$) \quad(\mathbf{s} \cdot \delta \mathbf{s}) \mathbf{T}=(\mathbf{T} \otimes \mathbf{s}) \delta \mathbf{s}, \quad(\mathbf{s} \cdot \delta \mathbf{s})(\mathbf{s} \times \mathbf{T})=-\mathbf{T} \times$ $[(\mathbf{s} \otimes \mathbf{s}) \delta \mathbf{s}],(\delta \mathbf{s} \times \mathbf{T})=-(\mathbf{T} \times \delta \mathbf{s})$ and resolving $\mathbf{t}$ in the middle surface basis $\left\{\mathbf{E}_{i}\right\}$, it follows that

$$
\begin{aligned}
\delta \mathbf{t}=\delta \hat{t}^{i} \mathbf{E}_{i}= & {\left[-\frac{\sin (\|\mathbf{s}\|)}{\|\mathbf{s}\|}\left[\begin{array}{cc}
0 & -1 \\
1 & 0 \\
\hat{s}^{1} & \hat{s}^{2}
\end{array}\right]\right.} \\
& \left.+\left(\frac{\sin (\|\mathbf{s}\|)}{\|\mathbf{s}\|^{3}}-\frac{\cos (\|\mathbf{s}\|)}{\|\mathbf{s}\|^{2}}\right)\left[\begin{array}{cc}
-\hat{s}^{2} \hat{s}^{1} & -\left(\hat{s}^{2}\right)^{2} \\
\left(\hat{s}^{1}\right)^{2} & \hat{s}^{1} \hat{s}^{2} \\
0 & 0
\end{array}\right]\right]\left\{\begin{array}{l}
\delta \hat{s}^{1} \\
\delta \hat{s}^{2}
\end{array}\right\}
\end{aligned}
$$

As already mentioned, all vectors in the computational process are defined relative to the standard basis $\mathbf{e}_{i}$. The transformation of the components $\delta \hat{t}^{i}$ is given by

$$
\left\{\begin{array}{l}
\delta t^{1} \\
\delta t^{2} \\
\delta t^{3}
\end{array}\right\}=\left[\begin{array}{ccc}
\Lambda_{11}^{0} & \Lambda_{12}^{0} & \Lambda_{13}^{0} \\
\Lambda_{21}^{0} & \Lambda_{22}^{0} & \Lambda_{23}^{0} \\
\Lambda_{31}^{0} & \Lambda_{32}^{0} & \Lambda_{33}^{0}
\end{array}\right]\left\{\begin{array}{l}
\delta \hat{t}^{1} \\
\delta \hat{t}^{2} \\
\delta \hat{t}^{3}
\end{array}\right\}
$$

\section{STRAIN MEASURES}

The Green-Lagrange strain tensor is given by standard expression

$$
\mathbf{E}=\frac{1}{2}\left(\mathbf{F}^{\mathrm{T}} \mathbf{F}-\mathbf{1}\right)
$$

Relative to the reference configuration contravariant basis, $\left\{\mathbf{G}^{i}\right\}_{i=1,2,3}$, the components of $\mathbf{E}$ are given by

$$
\mathbf{E}=\frac{1}{2}\left(\mathbf{g}_{i} \cdot \mathbf{g}_{j}-\mathbf{G}_{i} \cdot \mathbf{G}_{j}\right) \mathbf{G}^{i} \otimes \mathbf{G}^{j}=E_{i j} \mathbf{G}^{i} \otimes \mathbf{G}^{j}
$$

Using the definition of covariant base vectors, (6), and observing that $\mathbf{G}^{3}=\mathbf{T}$, the Green-Lagrangian strain tensor of the shell body may be obtained as

$$
\mathbf{E}=E_{\alpha \beta} \mathbf{G}^{\alpha} \otimes \mathbf{G}^{\beta}+E_{\alpha 3} \mathbf{G}^{\alpha} \otimes \mathbf{T}+E_{3 \alpha} \mathbf{T} \otimes \mathbf{G}^{\alpha}+E_{33} \mathbf{T} \otimes \mathbf{T}, \quad(\alpha=1,2)
$$

or equivalently

$$
\mathbf{E}=\mathbf{Z}^{-\mathbf{T}}\left(\mathbf{E}_{\alpha \beta} \mathbf{A}^{\alpha} \otimes \mathbf{A}^{\beta}+E_{\alpha 3} \mathbf{A}^{\alpha} \otimes \mathbf{T}+E_{3 \alpha} \mathbf{T} \otimes \mathbf{A}^{\alpha}+E_{33} \mathbf{T} \otimes \mathbf{T}\right) \mathbf{Z}^{-1}
$$

The components $E_{i j}$ may be written as functions of the through-thickness co-ordinate $\xi$

$$
E_{i j}=E_{i j}^{(0)}+\xi E_{i j}^{(1)}+(\xi)^{2} E_{i j}^{(2)}
$$


where

$$
\begin{aligned}
& E_{i j}^{(0)}=\frac{1}{2}\left(\mathbf{a}_{i} \cdot \mathbf{a}_{j}-\mathbf{A}_{i} \cdot \mathbf{A}_{j}\right) \\
& E_{\alpha \beta}^{(1)}=\frac{1}{2}\left(\mathbf{t}_{, \alpha} \cdot \mathbf{a}_{\beta}+\mathbf{t}_{, \beta} \cdot \mathbf{a}_{\alpha}-\mathbf{T}_{, \alpha} \cdot \mathbf{A}_{\beta}-\mathbf{T}_{, \beta} \cdot \mathbf{A}_{\alpha}\right) \\
& E_{\alpha 3}^{(1)}=E_{3 \alpha}^{(1)}=0 \\
& E_{33}^{(1)}=0 \\
& E_{\alpha \beta}^{(2)}=\frac{1}{2}\left(\mathbf{t}_{, \alpha} \cdot \mathbf{t}_{, \beta}-\mathbf{T}_{, \beta} \cdot \mathbf{T}_{, \beta}\right) \\
& E_{\alpha 3}^{(2)}=E_{3 \alpha}^{(2)}=0 \\
& E_{33}^{(2)}=0
\end{aligned}
$$

The base vectors of the tangent space to the deformed middle surface, $\mathbf{a}_{\alpha}$, may be decomposed as $\mathbf{a}_{\alpha}=\mathbf{A}_{\alpha}+\mathbf{u}_{, \alpha}\left(\mathbf{u}_{, \alpha}\right.$ denotes partial derivative of $\mathbf{u}$ with respect to $\left.\xi^{\alpha}\right)$. Consequently, the strain tensor components (39) may be rewritten in terms of the vector fields $\mathbf{u}$ and $\mathbf{t}$. The non-zero strain components are

$$
\begin{aligned}
& E_{\alpha \beta}^{(0)}=\frac{1}{2}\left(\mathbf{A}_{\alpha} \cdot \mathbf{u}_{, \beta}+\mathbf{A}_{\beta} \cdot \mathbf{u}_{, \alpha}+\mathbf{u}_{, \alpha} \cdot \mathbf{u}_{, \beta}\right) \\
& E_{\alpha 3}^{(0)}=E_{3 \alpha}^{(0)}=\frac{1}{2}\left(\mathbf{A}_{\alpha} \cdot(\mathbf{t}-\mathbf{T})+\mathbf{u}_{, \alpha} \cdot \mathbf{t}\right) \\
& E_{\alpha \beta}^{(1)}=\frac{1}{2}\left(\mathbf{A}_{\alpha} \cdot\left(\mathbf{t}_{, \beta}-\mathbf{T}_{, \beta}\right)+\mathbf{A}_{\beta} \cdot\left(\mathbf{t}_{, \alpha}-\mathbf{T}_{, \alpha}\right)+\mathbf{u}_{, \alpha} \cdot \mathbf{t}_{, \beta}+\mathbf{u}_{, \beta} \cdot \mathbf{t}_{, \alpha}\right) \\
& E_{\alpha \beta}^{(2)}=\frac{1}{2}\left(\mathbf{t}_{, \alpha} \cdot \mathbf{t}_{, \beta}-\mathbf{T}_{, \alpha} \cdot \mathbf{T}_{, \beta}\right)
\end{aligned}
$$

The above-defined strains and related numerical models have the following features:

(i) The linear variation of displacements through the shell thickness, (4), leads to quadratic distribution of the strain components $E_{\alpha \beta}$ through the shell thickness. The throughthickness distribution of the transverse shear strain $E_{\alpha 3}$ is constant.

(ii) The director field is, in this work, assumed to be inextensible. Computational models, based on Green-Lagrange strains, which explicitly include the through-thickness stretch were introduced by Simo et al. ${ }^{4}$ and Büchter and Ramm. ${ }^{18}$ In particular, Simo et al. consider uniform distribution in $\xi$-direction of the through-thickness stretch while Büchter and Ramm have recently included linear deformation of the director field via the so-called 'Enhanced Assumed Natural Strain' (EANS) formulation.

(iii) Within the inextensional director formulations the effect of change in the shell thickness due to the normal strains $E_{33}$ is usually obtained by enforcing the conditions of the zero through-thickness stress. This causes the condensation of the material law and gives quadratic deformation in the direction of the director for the St. Venant-Kirchhoff materials.

(iv) The three components of the axial vector relative to the standard basis $\left\{\mathbf{e}_{i}\right\}, \mathbf{s}=s^{i} \mathbf{e}_{i}$, are not independent, according to the constraints (18). It is therefore preferable to retain $\mathbf{s}$ in the local middle surface (Cartesian) basis $\mathbf{s}=\hat{s}^{1} \mathbf{E}_{1}+\hat{s}^{2} \mathbf{E}_{2}$, and obtain components of $\mathbf{t}$ in the standard basis, $\mathbf{t}=t^{i} \mathbf{e}_{i}$, by employing (23). Such an approach results in five unknown kinematic components, namely three components of $\mathbf{u}$ relative to the standard basis $\left\{\mathbf{e}_{i}\right\}$ and two components of $\mathbf{s}$ relative to the middle surface basis $\left\{\mathbf{E}_{i}\right\}$.

(v) The membrane strains, $E_{\alpha \beta}^{(0)}$, are not a function of rotational parameters, since the rotation about the middle surface normal $\mathbf{T}$ (also called a drill rotation) does not enter the 
formulation. When describing $\mathbf{t},(19)$, the rotation about the middle surface normal $\mathbf{T}$ is set to zero, which enables five kinematic fields to be retained. However, it is well-known that the analysis of non-smooth shells demands six kinematic fields. A computational approach which uses the above-defined strains is therefore restricted to the analysis of smooth shells. For a modification that enables the analysis of shells with intersections without changing the structure of the above equations see Reference 19.

(vi) Alternatively, one may, by resolving $\mathbf{s}$ in the standard basis, $\mathbf{s}=s^{i} \mathbf{e}_{i}$, define six kinematic variables (see Reference 5). Equation (20) may be used to obtain the components of $\mathbf{t}$ relative to $\left\{\mathbf{e}_{i}\right\}$. As already discussed such rotational components are not independent. Consequently, the rank deficiency in the element stiffness matrix is obtained, which equals the number of element nodes. For the shells with geometry approximated in a faceted form this does not create problems. However, stiffness matrix of a shell structure becomes singular if two neighbouring finite elements are coplanar.

\subsection{Variation of the strain measures}

Strain measure variations are obtained by taking the directional derivative in the direction of the virtual displacements, $\delta \mathbf{u}$, and virtual 'rotations', $\delta \mathbf{t}$, i.e. $(\delta \mathbf{u}, \delta \mathbf{t}):=\left.\frac{\mathrm{d}}{\mathrm{d} \varepsilon}\right|_{\varepsilon=0}\left(\boldsymbol{\varphi}_{\varepsilon}, \mathbf{t}_{\varepsilon}\right)$ which may be formally written as

$$
\delta \mathbf{E}(\mathbf{u}, \mathbf{t} ; \delta \mathbf{u}, \delta \mathbf{t}):=\left.\frac{\mathrm{d}}{\mathrm{d} \varepsilon}\right|_{\varepsilon=0} \mathbf{E}\left(\boldsymbol{\varphi}_{\varepsilon}, \mathbf{t}_{\varepsilon}\right)
$$

The resulting variations of strain measures may be expressed in the component form as $\left(\boldsymbol{\varphi}_{, \alpha}=\mathbf{a}_{\alpha}\right.$, see (9))

$$
\begin{aligned}
\delta E_{\alpha \beta}^{(0)}:=\mathrm{D}\left(E_{\alpha \beta}^{(0)}\right)[\delta \mathbf{u}, \delta \mathbf{t}]=\frac{1}{2}\left(\boldsymbol{\varphi}_{, \alpha} \cdot \delta \mathbf{u}_{, \beta}+\boldsymbol{\varphi}_{, \beta} \cdot \delta \mathbf{u}_{, \alpha}\right) \\
\delta E_{\alpha 3}^{(0)}:=\mathrm{D}\left(E_{\alpha 3}^{(0)}\right)[\delta \mathbf{u}, \delta \mathbf{t}]=\frac{1}{2}\left(\boldsymbol{\varphi}_{, \alpha} \cdot \delta \mathbf{t}+\delta \mathbf{u}_{, \alpha} \cdot \mathbf{t}\right) \\
\delta E_{\alpha \beta}^{(1)}:=\mathrm{D}\left(E_{\alpha \beta}^{(1)}\right)[\delta \mathbf{u}, \delta \mathbf{t}]=\frac{1}{2}\left(\boldsymbol{\varphi}_{, \alpha} \cdot \delta \mathbf{t}_{, \beta}+\boldsymbol{\varphi}_{, \beta} \cdot \delta \mathbf{t}_{, \alpha}+\delta \mathbf{u}_{, \alpha} \cdot \mathbf{t}_{, \beta}+\delta \mathbf{u}_{, \beta} \cdot \mathbf{t}_{, \alpha}\right) \\
\delta E_{\alpha \beta}^{(2)}:=\mathrm{D}\left(E_{\alpha \beta}^{(2)}\right)[\delta \mathbf{u}, \delta \mathbf{t}]=\frac{1}{2}\left(\delta \mathbf{t}_{, \alpha} \cdot \mathbf{t}_{, \beta}+\mathbf{t}_{, \alpha} \cdot \delta \mathbf{t}_{, \beta}\right)
\end{aligned}
$$

\section{PRINCIPLE OF VIRTUAL WORK}

The second Piola-Kirchhoff stress tensor of the three-dimensional shell body is defined as

$$
\mathbf{S}=S^{i j} \mathbf{G}_{i} \otimes \mathbf{G}_{j}
$$

By employing the definition of covariant base vectors (6), the stress tensor may be written as

$$
\mathbf{S}=S^{\alpha \beta} \mathbf{G}_{\alpha} \otimes \mathbf{G}_{\beta}+S^{\alpha 3} \mathbf{G}_{\alpha} \otimes \mathbf{T}+S^{3 \alpha} \mathbf{T} \otimes \mathbf{G}_{\alpha}+S^{33} \mathbf{T} \otimes \mathbf{T}
$$

or equivalently,

$$
\mathbf{S}=\mathbf{Z}\left(S^{\alpha \beta} \mathbf{A}_{\alpha} \otimes \mathbf{A}_{\beta}+S^{\alpha 3} \mathbf{A}_{\alpha} \otimes \mathbf{T}+S^{3 \alpha} \mathbf{T} \otimes \mathbf{A}_{\alpha}+S^{33} \mathbf{T} \otimes \mathbf{T}\right) \mathbf{Z}^{\mathrm{T}}
$$

The weak form of the equilibrium equations for the three-dimensional shell body takes the standard form

$$
G(\boldsymbol{\Phi} ; \delta \boldsymbol{\Phi})=G_{\text {int }}(\boldsymbol{\Phi} ; \delta \boldsymbol{\Phi})-G_{\text {ext }}(\delta \boldsymbol{\Phi})=\int_{\mathscr{B}} \mathbf{S}: \delta \mathbf{E}(\boldsymbol{\Phi} ; \delta \boldsymbol{\Phi}) \mathrm{d} V-\int_{\mathscr{B}} \mathbf{f} \cdot \delta \boldsymbol{\Phi} \mathrm{d} V-\int_{\partial \mathscr{B}} \overline{\mathbf{t}} \cdot \delta \boldsymbol{\Phi} \mathrm{d} S=0
$$


where $G_{\text {int }}(\boldsymbol{\Phi} ; \delta \boldsymbol{\Phi})$ and $G_{\text {ext }}(\delta \boldsymbol{\Phi})$ are commonly known as the internal and external virtual work, while $\mathbf{f}$ and $\overline{\mathbf{t}}$ denote the body and surface forces. The volume element, $\mathrm{d} V$, and the middle surface element, $\mathrm{d} A$, can be, in the convected co-ordinates, expressed by

$$
\mathrm{d} V=\sqrt{G} \mathrm{~d} \xi^{1} \mathrm{~d} \xi^{2} \mathrm{~d} \xi, \quad \mathrm{d} A=\sqrt{A} \mathrm{~d} \xi^{1} \mathrm{~d} \xi^{2}
$$

where $A=\operatorname{det}\left[A_{\alpha \beta}\right]$ and $G=\operatorname{det}\left[G_{\alpha \beta}\right]$. Furthermore, the following important relations are valid $\mathrm{d} V=\sqrt{G / A} \mathrm{~d} \xi \mathrm{d} A=\bar{\mu} \mathrm{d} \xi \mathrm{d} A$, where $\bar{\mu}=\sqrt{G / A}=\operatorname{det}[\mathbf{Z}]$.

By taking variations of the strain components, (39) and (40), the following expressions for the internal virtual work may be obtained:

$$
G_{\mathrm{int}}(\boldsymbol{\Phi} ; \delta \boldsymbol{\Phi})=\int_{\mathscr{M}}\left(\int_{h^{-}}^{h^{+}} S^{i j}\left(\delta E_{i j}^{(0)}+\xi \delta E_{i j}^{(1)}+(\xi)^{2} \delta E_{i j}^{(2)}\right) \bar{\mu} \mathrm{d} \xi\right) \mathrm{d} A,
$$

where $h^{-}$and $h^{+}$denote co-ordinates of the bottom and top surfaces of the shell, respectively. The above expression motivates definition of the second Piola-Kirchhoff stress resultant tensors

$$
\begin{gathered}
\overline{\mathbf{n}}=n^{i j} \mathbf{G}_{i} \otimes \mathbf{G}_{j}, \quad n^{i j}=\int_{h^{-}}^{h^{+}} S^{i j} \bar{\mu} \mathrm{d} \xi \\
\overline{\mathbf{m}}^{(1)}=m^{i j(1)} \mathbf{G}_{i} \otimes \mathbf{G}_{j}, \quad m^{i j(1)}=\int_{h^{-}}^{h^{+}} S^{i j} \xi \bar{\mu} \mathrm{d} \xi \\
\overline{\mathbf{m}}^{(2)}=m^{i j(2)} \mathbf{G}_{i} \otimes \mathbf{G}_{j}, \quad m^{i j(2)}=\int_{h^{-}}^{h^{+}} S^{i j}(\xi)^{2} \bar{\mu} \mathrm{d} \xi
\end{gathered}
$$

The stress resultant tensors (49)-(51) are symmetric, opposite to their physical counterparts (see References 11 and 20). Since they take part in the weak form of the equations of equilibrium, they are also called the effective stress resultant tensors. Inserting the definition of the second Piola-Kirchhoff stress resultants into (48) the two-dimensional expression for the internal virtual work may be obtained. A similar procedure which leads to the two-dimensional external virtual work will not be discussed here (see e.g. Reference 7).

The resulting two-dimensional expression for the principle of virutal work

$$
G(\boldsymbol{\Phi} ; \delta \boldsymbol{\Phi})=\int_{\mathscr{M}}\left(n^{i j} \delta E_{i j}^{(0)}+m^{i j(1)} \delta E_{i j}^{(1)}+m^{i j(2)} \delta E_{i j}^{(2)}\right) \mathrm{d} A-G_{\text {ext }}(\delta \boldsymbol{\Phi})
$$

is a governing equation for the displacement-based finite element formulation.

\section{ELASTO-PLASTIC CONSTITUTIVE MODEL}

In this section, formulation and computational aspects of the elasto-plastic model for thin shells are described. A simple von Mises yield criterion is adopted with isotropic hardening. Attention is restricted to situations where strains remain infinitesimal, although rotations are not restricted. Details and motivation for the present constitutive description may be found in Reference 1, while numerical treatment follows References 2 and 3.

\subsection{Hyperelastic stress response}

6.1.1. Three-dimensional hyperelastic model. Assume that the constitutive response is governed by a stored-energy function $W(\mathbf{E}, \mathbf{X})$. The simplest among the non-linear hyperelastic, homogene- 
ous, isotropic material models is the so-called St. Venant-Kirchhoff material. For this material the stored-energy function takes the form

$$
W(\mathbf{E})=\frac{\lambda}{2}(\operatorname{tr}[\mathbf{E}])^{2}+\mu \operatorname{tr}\left[\mathbf{E}^{2}\right]
$$

where $\lambda$ and $\mu$ are Lamé material parameters expressed with Young's modulus $E$ and Poisson's coefficient $v$ as

$$
\lambda=\frac{E v}{(1+v)(1-2 v)}, \quad \mu=\frac{E}{2(1+v)}
$$

The stresses in the shell body are given by

$$
\mathbf{S}=\frac{\partial W(\mathbf{E})}{\partial \mathbf{E}}=\lambda \operatorname{tr}[\mathbf{E}] \mathbf{1}+2 \mu \mathbf{E}=\mathbf{H}[\mathbf{E}]
$$

where $\mathbf{H}$ is the fourth-order isotropic tensor. Equation (55) may be written in the component form (by employing (35) and (43)) as

$$
\begin{aligned}
S^{i j} & =\left(\lambda G^{i j} G^{k l}+\mu\left(G^{i k} G^{j l}+G^{i l} G^{j k}\right)\right) E_{k l} \\
& =\mathrm{H}^{i j k l} E_{k l}=\mathrm{H}^{i j k l}\left(E_{k l}^{(0)}+\xi E_{k l}^{(1)}+(\xi)^{2} E_{k l}^{(2)}\right)
\end{aligned}
$$

6.1.2. Thin-shell hyperelastic model. A thin-shell hyperelastic constitutive model follows from (56) by enforcing the zero through-thickness stress, i.e.

$$
S^{33}=\mathrm{H}^{33 k l}\left(E_{k l}^{(0)}+\xi E_{k l}^{(1)}+(\xi)^{2} E_{k l}^{(2)}\right)+\mathrm{H}^{3333} E_{33}=0, \quad k l \neq 33
$$

Accordingly, the constitutive law may be condensed resulting in

$$
\mathrm{C}^{i j k l}=\mathrm{H}^{i j k l}-\frac{\mathrm{H}^{33 k l}}{\mathrm{H}^{3333}}, \quad i j \neq 33, k l \neq 33
$$

This leads to

$$
\begin{aligned}
n^{i j} & =\int_{h^{-}}^{h^{+}} \mathrm{C}^{i j k l}\left(E_{k l}^{(0)}+\xi E_{k l}^{(1)}+(\xi)^{2} E_{k l}^{(2)}\right) \bar{\mu} \mathrm{d} \xi, \quad i j \neq 33, k l \neq 33 \\
m^{i j(1)} & =\int_{h^{-}}^{h^{+}} \mathrm{C}^{i j k l}\left(E_{k l}^{(0)}+\xi E_{k l}^{(1)}+(\xi)^{2} E_{k l}^{(2)}\right) \xi \bar{\mu} \mathrm{d} \xi, \quad i j \neq 33, k l \neq 33 \\
m^{i j(2)} & =\int_{h^{-}}^{h^{+}} \mathrm{C}^{i j k l}\left(E_{k l}^{(0)}+\xi E_{k l}^{(1)}+(\xi)^{2} E_{k l}^{(2)}\right)(\xi)^{2} \bar{\mu} \mathrm{d} \xi, \quad i j \neq 33, k l \neq 33
\end{aligned}
$$

The above expressions can be equivalently written in the form

$$
\left\{\begin{array}{c}
n^{i j} \\
m^{i j(1)} \\
m^{i j(2)}
\end{array}\right\}=\left[\begin{array}{lll}
\mathrm{C}_{0}^{i j k l} & \mathrm{C}_{1}^{i j k l} & \mathrm{C}_{2}^{i j k l} \\
\mathrm{C}_{1}^{i j k l} & \mathrm{C}_{2}^{i j k l} & \mathrm{C}_{3}^{i j k l} \\
\mathrm{C}_{2}^{i j k l} & \mathrm{C}_{3}^{i j k l} & \mathrm{C}_{4}^{i j k l}
\end{array}\right]\left\{\begin{array}{c}
E_{k l}^{(0)} \\
E_{k l}^{(1)} \\
E_{k l}^{(2)}
\end{array}\right\}, \quad i j \neq 33, k l \neq 33
$$

where

$$
\mathrm{C}_{J}^{i j k l}=\int_{h^{-}}^{h^{+}} \mathrm{C}^{i j k l}(\xi)^{J} \bar{\mu} \mathrm{d} \xi, \quad J=0,1,2,3,4, \quad i j \neq 33, k l \neq 33
$$


From equation (57) it follows that, for the St. Venant-Kirchhoff material, the strain $E^{33}$ becomes a quadratic function of $\xi$.

\subsection{Three-dimensional elasto-plastic constitutive model}

6.2.1. Kinematic description of elasto-plastic deformations. In the description of the present three-dimensional elasto-plastic constitutive model, attention is restricted to situations where strain tensor remains small. In this case, the total strain tensor may be additively decomposed* into elastic and plastic part, i.e.

$$
\mathbf{E}=\mathbf{E}^{\mathrm{e}}+\mathbf{E}^{\mathrm{p}}
$$

6.2.2. Thermodynamical basis. A broad framework for analysis of three-dimensional elastoplastic solids is provided by the thermodynamic theory with internal variables. ${ }^{1}$ In this work, attention is restricted to isothermal processes, while, for the present purpose, it suffices to assume that the plastic process is described by a single scalar variable representing the isotropic hardening. Then, a thermodynamic state at a point under consideration is fully described by a set $\left\{\mathbf{E}^{\mathrm{e}}, R\right\}$, where $\mathbf{E}^{\mathrm{e}}$ is the elastic part of the Green-Lagrange strain tensor, while $R$ is a scalar which describes the isotropic hardening.

By assuming that the elastic response of the material and plastic hardening are decoupled, the specific free energy (which, for isothermal processes, equals the specific deformation energy $W=W\left(\mathbf{E}^{\mathrm{e}}, R\right)$ may be represented in the additive form as

$$
W\left(\mathbf{E}^{\mathrm{e}}, R\right)=W^{\mathrm{e}}\left(\mathbf{E}^{\mathrm{e}}\right)+W^{\mathrm{p}}(R)
$$

Recall that the second Piola-Kirchhoff stress tensor $\mathbf{S}$ is energy conjugate to the Green-Lagrange strain tensor E. The second law of thermodynamics in the form of Clausius-Duhem inequality requires ${ }^{1}$

$$
\mathbf{S}: \frac{\mathrm{d} \mathbf{E}}{\mathrm{d} t}-\frac{\mathrm{d} W}{\mathrm{~d} t} \geqslant 0
$$

By employing the specific deformation energy (62), the additive decomposition (61) and assuming that the elastic behaviour is isotropic it follows that

$$
\left(\mathbf{S}-\frac{\partial W^{\mathrm{e}}}{\partial \mathbf{E}^{\mathrm{e}}}\right): \dot{\mathbf{E}}^{\mathrm{e}}+\mathbf{S}: \dot{\mathbf{E}}^{\mathrm{p}}-K \dot{R} \geqslant 0
$$

where

$$
K=\frac{\partial W^{\mathrm{p}}}{\partial R}
$$

is a thermodynamic force conjugate to $R$, and $(\cdot)$ denotes $\mathrm{d}(\bullet) / \mathrm{d} t$. For purely elastic process it follows that $\dot{\mathbf{E}}^{\mathrm{p}}=0$ and $\dot{R}=0$. Since the Clausius-Duhem inequality is valid for any $\dot{\mathbf{E}}^{\mathrm{e}}$, from (63b) it follows that

$$
\mathbf{S}=\frac{\partial W^{\mathrm{e}}}{\partial \mathbf{E}^{\mathrm{e}}}
$$

\footnotetext{
* For large deformations of elasto-plastic solids at finite strains an alternative, and recently very popular, formulation is possible based on the multiplicative decomposition of the total deformation gradient $\mathbf{F}$ into elastic and plastic parts, $\mathbf{F}=\mathbf{F}^{\mathrm{e}} \mathbf{F}^{\mathrm{p}}$ (see Reference 21$)$
} 
The elastic part of the specific energy is assumed to be fully described by the St. VenantKirchhoff model given in Section 6.1.

6.2.3. Yield criterion and loading/unloading conditions. In correspondence with the standard theory of plasticity, existence of the yield surface in the stress space is assumed in the form $\Phi=\Phi(\mathbf{S}, K)$. The admissible stresses can never leave the closed domain specified by

$$
\Phi(\mathbf{S}, K) \leqslant 0
$$

At some time instant $t \in[0, T]$ for the process to be elastic it is required that $\Phi(\mathbf{S}, K)<0$ and $\dot{\mathbf{E}}^{\mathrm{p}}=0, \dot{R}=0$, while for plastic deformations to be present it is necessary that $\Phi(\mathbf{S}, K)=0$. (If $\mathrm{d} \Phi>0$ the loading is taking place, $\mathrm{d} \Phi=0$ specifies the neutral loading while $\mathrm{d} \Phi<0$ specifies the elastic unloading.)

The associative theories describe a broad class of elasto-plastic material models which are characterised by the so-called normality principle. For these materials plastic flow and hardening rule may be represented in the form ${ }^{1}$

$$
\begin{aligned}
\dot{\mathbf{E}}^{\mathrm{p}} & =\dot{\lambda} \frac{\partial \Phi}{\partial \mathbf{S}} \\
\dot{R} & =-\dot{\lambda} \frac{\partial \Phi}{\partial K}
\end{aligned}
$$

The so-called consistency parameter $\dot{\lambda} \geqslant 0$ is consistent with the loading and unloading which are expressed as the Kuhn-Tucker conditions ${ }^{2}$

$$
\Phi \leqslant 0, \quad \dot{\lambda} \geqslant 0, \quad \dot{\lambda} \Phi=0
$$

These conditions must be satisfied at any time instant $t \in[0, T]$.

6.2.4. Von Mises plasticity model-small-strain description. Elasto-plastic material considered in this work will be assumed to follow the von Mises plasticity model. The yield criterion will be represented in the form

$$
\Phi(\mathbf{S}, K)=\frac{1}{2}\left\|\mathbf{S}^{\prime}\right\|^{2}-\frac{1}{3}\left(S_{\mathrm{Y}}+K\right)^{2} \leqslant 0
$$

where $\mathbf{S}^{\prime}:=\operatorname{dev}[\mathbf{S}]=\mathbf{S}-\frac{1}{3} \operatorname{tr}[\mathbf{S}]$ is the deviatoric stress tensor, $S_{\mathrm{Y}}$ is the uniaxial yield stress and $\|\operatorname{dev}[\mathbf{S}]\|:=\left(\mathbf{S}^{\prime}: \mathbf{S}^{\prime}\right)^{1 / 2}=\left(S_{i j}^{\prime} S_{i j}^{\prime}\right)^{1 / 2}$.

The associated flow rule and hardening rule will be considered which for the von Mises yield criterion (67) may be expressed as

$$
\begin{aligned}
\dot{\mathbf{E}}^{\mathrm{p}} & =\dot{\lambda} \frac{\partial \Phi(\mathbf{S}, K)}{\partial \mathbf{S}}=\dot{\lambda} \mathbf{S}^{\prime} \\
\dot{K} & =-\dot{\lambda} K^{\prime}(R) \frac{\partial \Phi(\mathbf{S}, K)}{\partial K}=\dot{\lambda} \frac{2}{3} K^{\prime}(R)\left[S_{\mathrm{Y}}+K(R)\right] \\
\dot{R} & =\left[K^{\prime}(R)\right]^{-1} \dot{K}
\end{aligned}
$$

Equations (68) further imply the following important relationships:

$$
\dot{\lambda}=\left\|\dot{\mathbf{E}}^{\mathrm{p}}\right\|\left\|\mathbf{S}^{\prime}\right\|^{-1}
$$




$$
R(t)=\int_{0}^{t} \sqrt{\frac{2}{3}}\left\|\dot{\mathbf{E}}^{\mathrm{p}}(\tau)\right\| \mathrm{d} \tau
$$

Relation (69b) defines the internal variable $R(t)$ as the so-called equivalent plastic strain.

Remark 3. We note that in the case of the full finite strain kinematics the correct definition of the deviatoric part of the second Piola-Kirchhoff stress tensor is

$$
\mathbf{S}^{\prime}=\operatorname{Dev}[\mathbf{S}]=\mathbf{S}-\frac{1}{3}[\mathbf{S}: \mathbf{C}] \mathbf{C}^{-1}
$$

Since $\mathbf{C}=(\mathbf{1}+2 \mathbf{E})$, then for small Green-Lagrange strains (i.e. when $\left\|\mathbf{E}^{2}\right\| \ll\|\mathbf{E}\| \ll 1$ ) it follows that

$$
\mathbf{S}^{\prime}=\mathbf{S}-\frac{1}{3}[\mathbf{S}: \mathbf{1}] \mathbf{1}+\mathcal{O}(\|\mathbf{E}\|)=\operatorname{dev}[\mathbf{S}]+\mathcal{O}(\|\mathbf{E}\|)
$$

where $\mathcal{O}(\|\mathbf{E}\|)$ denotes the terms which tend to zero, i.e. $\mathcal{O}(\|\mathbf{E}\|) \rightarrow 0$, as $\|\mathbf{E}\| \rightarrow 0$. Thus, when strains are restricted to be small, an approximate definition of the deviatoric stress tensor may be employed. Therefore, in problems where finite rotations may be present but strains are restricted to be small, the above von Mises model for large deformations of elasto-plastic shells (67)-(69) is equivalent to the small-strain von Mises elasto-plastic model. Accordingly, all aspects of material identification known for small-strain elasto-plastic models transfer immediately to the present elasto-plastic thin-shell model. For situations arising during failures of elasto-plastic thin shells typically associated with buckling-type instabilities and/or bending-dominated shell problems resulting in the formation of plastic hinges this model is expected to provide accurate description of the physical phenomena.

\subsection{Elasto-plastic constitutive model for thin shells: matrix formulation}

The present elasto-plastic constitutive model for thin shells employs the condition of the zero stress in normal direction, i.e. $S^{33}=0$. Hence, in matrix formulation of the elasto-plastic constitutive model the following definitions will be employed: ${ }^{\dagger}$

$$
\begin{aligned}
\mathbf{S}^{\mathrm{T}} & =\left\lfloor S^{11}, S^{22}, S^{12}, S^{13}, S^{23}\right\rfloor_{(1 \times 5)} \\
\mathbf{S}^{\prime \mathrm{T}} & =\left\lfloor S^{\prime 11}, S^{\prime 22}, S^{\prime 12}, S^{\prime 13}, S^{\prime 23}\right\rfloor_{(1 \times 5)} \\
\mathbf{E}^{\mathrm{T}} & =\left\lfloor E_{11}, E_{22}, 2 E_{12}, 2 E_{13}, 2 E_{23}\right\rfloor_{(1 \times 5)} \\
\left(\mathbf{E}^{\mathrm{p}}\right)^{\mathrm{T}} & =\left\lfloor E_{11}^{\mathrm{p}}, E_{22}^{\mathrm{p}}, 2 E_{12}^{\mathrm{p}}, 2 E_{13}^{\mathrm{p}}, 2 E_{23}^{\mathrm{p}}\right\rfloor_{(1 \times 5)}
\end{aligned}
$$

Here $\mathbf{S}$ denotes the stress vector, $\mathbf{S}^{\prime}$ is the deviatoric stress vector, $\mathbf{E}$ is the strain vector and $\mathbf{E}^{\mathrm{p}}$ is the plastic strain vector. The vectors (72a)-(72d) consist of the physical components of stresses and strains, i.e. they are defined relative to the orthonormal basis, opposite to the stress-strain components defined earlier in equations (35), (43) and Section 6.1. This approach considerably simplifies the implementation of elasto-plastic constitutive law. In addition, the following matrix relationship will be used

$$
\mathbf{S}^{\prime}=\mathbf{P}_{1} \mathbf{S}
$$

\footnotetext{
${ }^{\dagger}$ In the following $\mathbf{S}, \mathbf{S}^{\prime}, \mathbf{E}, \mathbf{E}^{\mathrm{e}}$ and $\mathbf{E}^{\mathrm{p}}$ will denote the corresponding vectors while, in Section 6.2, the same notation was related to the corresponding tensors
} 
where the matrix $\mathbf{P}_{1}$ may be expressed as

$$
\mathbf{P}_{1}=\frac{1}{3}\left[\begin{array}{rrrrr}
2 & -1 & 0 & 0 & 0 \\
-1 & 2 & 0 & 0 & 0 \\
0 & 0 & 3 & 0 & 0 \\
0 & 0 & 0 & 3 & 0 \\
0 & 0 & 0 & 0 & 3
\end{array}\right]_{(5 \times 5)}
$$

In accordance with the model described in Section 6.2, notation (72), the elasto-plastic constitutive model for thin shells may be written in the following matrix form:

$$
\begin{aligned}
\mathbf{E} & =\mathbf{E}^{\mathrm{e}}+\mathbf{E}^{\mathrm{p}} \\
\mathbf{S} & =\mathbf{C}\left[\mathbf{E}^{\mathrm{e}}\right] \\
\Phi & =\frac{1}{2} \mathbf{S}^{\mathrm{T}} \mathbf{P}_{2} \mathbf{S}-\frac{1}{3}\left[S_{\mathrm{Y}}+K\left(\bar{E}^{\mathrm{p}}\right)\right]^{2} \\
\dot{\mathbf{E}}^{\mathrm{p}} & =\dot{\lambda} \mathbf{P}_{2} \mathbf{S} \\
\dot{\bar{E}}^{\mathrm{p}} & =\dot{R}=\dot{\lambda}\left(\frac{2}{3} \mathbf{S}^{\mathrm{T}} \mathbf{P}_{2} \mathbf{S}\right)^{1 / 2}
\end{aligned}
$$

with loading/unloading conditions expressed as

$$
\Phi \leqslant 0, \quad \dot{\lambda} \geqslant 0, \quad \dot{\lambda} \Phi=0
$$

Matrix $\mathbf{P}_{2}$ has the form

$$
\mathbf{P}_{2}=\frac{1}{3}\left[\begin{array}{rrrrr}
2 & -1 & 0 & 0 & 0 \\
-1 & 2 & 0 & 0 & 0 \\
0 & 0 & 6 & 0 & 0 \\
0 & 0 & 0 & 6 & 0 \\
0 & 0 & 0 & 0 & 6
\end{array}\right]_{(5 \times 5)}
$$

Note that matrix $\mathbf{P}_{2}$ differs from the earlier matrix $\mathbf{P}_{1}$ as a result of the effect of shear terms of $\dot{\mathbf{E}}^{\text {p }}$ in (74d) and usage of $S^{\prime 33}$ in (74c), e.g. for shear component of $\dot{\mathbf{E}}^{\mathrm{p}}$ it follows $2 \dot{E}_{12}^{\mathrm{p}}=\dot{\lambda} 2{S^{\prime}}^{12}$. Matrix C is the matrix of elastic constants for the thin shells obtained by condensation $\left(S^{33}=0\right)$ of the three-dimensional relations (see Section 6.1). Since the orthonormal co-ordinate system is adopted in this section, form of the elastic constitutive matrix is simpler than the one that may be obtained from equation (56). The shear correction factors are taken as equal to 1.

Observe that $E_{33}^{\mathrm{e}}$ and $E_{33}^{\mathrm{p}}$ are not zero, which is not explicitly stated in the above equations.

\subsection{Integration algorithm for thin shell elasto-plastic constitutive model}

In the solution of the non-linear evolution problem for the rate-independent thin-shell elastoplasticity, described in Sections 6.2 and 6.3, a numerical time integration procedure is typically employed, which provides an approximate solution to the problem at discrete points of the time interval $[0, T]=\bigcup_{n=1}^{N}\left[t_{n}, t_{n+1}\right]$. In this section, the so-called generalized midpoint algorithm, formalized by Ortiz and Popov, ${ }^{22}$ is employed.

6.4.1. Problem definition. The equilibrium equations are assumed to be satisfied at time instant $t_{n}$. In addition, it is assumed that the stress field $\mathbf{S}_{n}$ and the internal variables $\left\{\mathbf{E}_{n}^{\mathrm{p}}, \bar{E}_{n}^{\mathrm{p}}\right\}$ satisfy the discrete version of the constitutive law described in Sections 6.2 and 6.3. For the present purpose, 
it may also be assumed that the displacement field $\mathbf{u}_{n+1}$ and hence, the strain field $\mathbf{E}_{n+1}$ are given at the time instant $t_{n+1}$. The problem consists in evaluating the stress field $\mathbf{S}_{n+1}$ and the set of internal variables $\left\{\mathbf{E}_{n+1}^{\mathbf{p}}, \bar{E}_{n+1}^{\mathbf{p}}\right\}$ at the time instant $t_{n+1}$ which are consistent with the discrete version of the constitutive law described in Sections 6.2 and 6.3.

6.4.2. Integration algorithm. By employing the generalized midpoint algorithm to the evolution problem of thin-shell rate-independent elasto-plasticity described in Section 6.3, the incremental version of the elasto-plastic evolution problem is obtained as follows: Values of $\mathbf{E}_{n+1}^{\mathrm{p}}$ and $\bar{E}_{n+1}^{\mathrm{p}}$ at the time instant $t_{n+1}$ are obtained as

$$
\begin{aligned}
& \mathbf{E}_{n+1}^{\mathrm{p}}=\mathbf{E}_{n}^{\mathrm{p}}+\Delta \mathbf{E}_{n+\alpha}^{\mathrm{p}} \\
& \bar{E}_{n+1}^{\mathrm{p}}=\bar{E}_{n}^{\mathrm{p}}+\Delta \bar{E}_{n+\alpha}^{\mathrm{p}}
\end{aligned}
$$

where $t_{n+\alpha} \in\left[t_{n}, t_{n+1}\right], \alpha \in[0,1]$ and

$$
\begin{aligned}
\Delta \mathbf{E}_{n+\alpha}^{\mathrm{p}} & =\frac{1}{\alpha}\left(\mathbf{E}_{n+\alpha}^{\mathrm{p}}-\mathbf{E}_{n}^{\mathrm{p}}\right) \\
\Delta \bar{E}_{n+\alpha}^{\mathrm{p}} & =\frac{1}{\alpha}\left(\bar{E}_{n+\alpha}^{\mathrm{p}}-\bar{E}_{n}^{\mathrm{p}}\right)
\end{aligned}
$$

Values at the time instant $t_{n+\alpha}$ are obtained as

$$
\begin{aligned}
\mathbf{E}_{n+\alpha} & =\mathbf{E}_{n}+\alpha \Delta \mathbf{E}_{n}=(1-\alpha) \mathbf{E}_{n}+\alpha \mathbf{E}_{n+1} \\
\mathbf{E}_{n+\alpha}^{\mathrm{p}} & =\mathbf{E}_{n}^{\mathrm{p}}+\alpha \Delta \mathbf{E}_{n}^{\mathrm{p}}=(1-\alpha) \mathbf{E}_{n}^{\mathrm{p}}+\alpha \mathbf{E}_{n+1}^{\mathrm{p}} \\
\bar{E}_{n+\alpha}^{\mathrm{p}} & =\bar{E}_{n}^{\mathrm{p}}+\alpha \Delta \bar{E}_{n}^{\mathrm{p}}=(1-\alpha) \bar{E}_{n}^{\mathrm{p}}+\alpha \bar{E}_{n+1}^{\mathrm{p}} \\
\mathbf{S}_{n+\alpha} & =\mathbf{C}\left[\mathbf{E}_{n+\alpha}-\mathbf{E}_{n+\alpha}^{\mathrm{p}}\right]
\end{aligned}
$$

In accordance with equations (75)-(77) it follows that

$$
\begin{gathered}
\mathbf{E}_{n+1}^{\mathrm{p}}=\mathbf{E}_{n}^{\mathrm{p}}+\lambda_{n+\alpha} \mathbf{P}_{2} \mathbf{S}_{n+\alpha} \\
\bar{E}_{n+1}^{\mathrm{p}}=\bar{E}_{n}^{\mathrm{p}}+\lambda_{n+\alpha} \sqrt{\frac{2}{3}}\left(\mathbf{S}_{n+\alpha}^{\mathrm{T}} \mathbf{P}_{2} \mathbf{S}_{n+\alpha}\right)^{1 / 2}
\end{gathered}
$$

where the notation $\lambda_{n+\alpha}=\Delta t \dot{\lambda}$ is introduced.

The incremental elasto-plastic problem (75)-(78), is solved by employing the standard operator split methodology, where the original problem, (75)-(78) is solved through composition, applying first the elastic and then the plastic algorithm. The essential steps of this well-known numerical procedure are briefly summarized in Box 1, while details of implementation with explicit expressions may be found in References 2 and 3.

\subsection{Consistent elasto-plastic tangent modulus}

In agreement with notation introduced in Section 5, the weak form of equilibrium for a shell may be expressed as

$$
G\left(\boldsymbol{\Phi}_{n+1}, \delta \boldsymbol{\Phi}\right)=G_{\text {int }}\left(\boldsymbol{\Phi}_{n+1}, \delta \boldsymbol{\Phi}\right)-G_{\text {ext }}(\delta \boldsymbol{\Phi})=0
$$

where $G_{\text {int }}\left(\boldsymbol{\Phi}_{n+1}, \delta \boldsymbol{\Phi}\right)$ and $G_{\text {ext }}(\delta \boldsymbol{\Phi})$ represent the internal and external virtual work, respectively (see (46)). With known state at $t_{n}$ defined by $\left\{\mathbf{E}_{n}, \mathbf{E}_{n}^{\mathrm{p}}, \bar{E}_{n}^{\mathrm{p}}\right\}$, and given external loading at $t_{n+1}$, a new configuration is required $\boldsymbol{\Phi}_{n+1}:=\boldsymbol{\Phi}\left(t_{n+1}\right)$ and internal variables $\left\{\mathbf{E}_{n+1}^{\mathbf{p}}, \bar{E}_{n+1}^{\mathbf{p}}\right\}$ consistent 
Box 1. Elastic predictor - plastic corrector algorithm for the von-Mises thin shell elasto-plastic constitutive model

(i) Evaluate elastic predictor

$$
\begin{aligned}
\mathbf{E}_{n+\alpha} & =(1-\alpha) \mathbf{E}_{n}+\alpha \mathbf{E}_{n+1} \\
\mathbf{S}_{n+\alpha}^{\text {trial }} & =\mathbf{C}\left[\mathbf{E}_{n+\alpha}-\mathbf{E}_{n+\alpha}^{\text {p,trial }}\right]=\mathbf{C}\left[\mathbf{E}_{n+\alpha}-\mathbf{E}_{n}^{\mathrm{p}}\right]
\end{aligned}
$$

(ii) Check yield condition

$$
\begin{aligned}
& \Phi_{n+\alpha}^{\text {trial }}=\frac{1}{2}\left(\mathbf{S}_{n+\alpha}^{\text {trial }}\right)^{\mathrm{T}} \mathbf{P}_{2} \mathbf{S}_{n+\alpha}^{\text {trial }}-\frac{1}{3}\left[S_{\mathrm{Y}}+K\left(\bar{E}_{n+\alpha}^{\mathrm{p}, \text { trial }}\right)\right]^{2} \\
& \text { If } \Phi_{n+\alpha}^{\text {trial }} \leqslant 0 \quad \text { THEN } \\
& \text { (Elastic State) } \\
& \text { Set }(\bullet)_{n+\alpha}=(\bullet)_{n+\alpha}^{\text {trial }} \leqslant 0 \text { and GOTO (iii.2) } \\
& \text { ELSE } \\
& \text { GOTO (iii) }
\end{aligned}
$$

(iii) Evaluate the plastic corrector

(iii.1) Evaluate the consistency parameter $\lambda_{n+\alpha}$ by $\mathrm{N}-\mathrm{R}$ solution of:

$$
\begin{aligned}
& \Phi_{n+\alpha}=\frac{1}{2} \mathbf{S}_{n+\alpha}^{\mathrm{T}} \mathbf{P}_{2} \mathbf{S}_{n+\alpha}-\frac{1}{3}\left[S_{\mathrm{Y}}+K\left(\bar{E}_{n+\alpha}^{\mathrm{p}}\right)\right]^{2}=0 \\
& \bar{E}_{n+\alpha}^{\mathrm{p}}=\bar{E}_{n}^{\mathrm{p}}+\alpha \lambda_{n+\alpha} \sqrt{\frac{2}{3}}\left(\mathbf{S}_{n+\alpha}^{\mathrm{T}} \mathbf{P}_{2} \mathbf{S}_{n+\alpha}\right)^{1 / 2} .
\end{aligned}
$$

(iii.2) Update stresses, plastic strain and hardening parameter

$$
\begin{aligned}
\hat{\mathbf{C}} & =\left(\mathbf{I}+\alpha \lambda_{n+\alpha} \mathbf{C P}_{2}\right)^{-1} \mathbf{C}=\left(\mathbf{C}^{-1}+\alpha \lambda_{n+\alpha} \mathbf{P}_{2}\right)^{-1} \\
\mathbf{S}_{n+\alpha} & =\hat{\mathbf{C}}\left[\mathbf{E}_{n+\alpha}-\mathbf{E}_{n}^{\mathrm{p}}\right] \\
\mathbf{E}_{n+1}^{\mathrm{p}} & =\mathbf{E}_{n}^{\mathrm{p}}+\lambda_{n+\alpha} \mathbf{P}_{2} \mathbf{S}_{n+\alpha} \\
\bar{E}_{n+1}^{\mathrm{p}} & =\bar{E}_{n}^{\mathrm{p}}+\lambda_{n+\alpha} \sqrt{\frac{2}{3}}\left[\mathbf{S}_{n+\alpha}^{\mathrm{T}} \mathbf{P}_{2} \mathbf{S}_{n+\alpha}\right]^{1 / 2} \\
K_{n+1} & =K\left(\bar{E}_{n+1}^{\mathrm{p}}\right) \\
\mathbf{S}_{n+1} & =\hat{\mathbf{C}}\left[\mathbf{E}_{n+1}-\mathbf{E}_{n+1}^{\mathrm{p}}\right]
\end{aligned}
$$

with the discrete constitutive model described in Section 6.4. This procedure defines an (algorithmic) incremental relationship

$$
\mathbf{S}_{n+1}=\tilde{\mathbf{S}}\left(\boldsymbol{\Phi}_{n+1}, \mathbf{E}_{n+1}^{\mathrm{p}}, \bar{E}_{n+1}^{\mathrm{p}} ; \mathbf{E}_{n}^{\mathrm{p}}, \bar{E}_{n}^{\mathrm{p}}\right)
$$

Incremental problem may then be defined as: For the known state $\left\{\mathbf{E}_{n}, \mathbf{E}_{n}^{\mathrm{p}}, \bar{E}_{n}^{\mathrm{p}}\right\}$ and known loading at $t_{n+1}$ find the configuration $\boldsymbol{\Phi}_{n+1}$, and the state variables $\left\{\mathbf{E}_{n+1}^{\mathrm{p}}, \bar{E}_{n+1}^{\mathrm{p}}\right\}$, which satisfy weak equilibrium statement (79) and are consistent with the constitutive model described in Section 6.4.

Equation (79) defines a highly non-linear function and requires, in general, an iterative solution procedure. In this work, the solution is being sought by using the Newton-Raphson procedure. As will be detailed in Section 9, linearization of the incremental boundary value problem at the given configuration $\boldsymbol{\Phi}_{n+1}$ is required, which also involves, as its essential part, the linearization of the constitutive equations at the fixed geometry.

The linearized form of the relationship (80) - i.e. the derivative

$$
\overline{\mathbf{C}}_{n+1}=\frac{\partial \mathbf{S}_{n+1}}{\partial \mathbf{E}_{n+1}}
$$


which defines the elasto-plastic modulus $\overline{\mathbf{C}}_{n+1}$ consistent with applied time integration algorithm - can be evaluated in the closed form as (see References 2 and 3)

$$
\overline{\mathbf{C}}_{n+1}=\hat{\mathbf{C}}-\frac{\hat{\mathbf{C}} \mathbf{P}_{2} \mathbf{S}_{n+\alpha} \mathbf{S}_{n+\alpha}^{\mathrm{T}} \mathbf{P}_{2} \hat{\mathbf{C}}}{\mathbf{S}_{n+\alpha}^{\mathrm{T}} \mathbf{P}_{2} \hat{\mathbf{C}} \mathbf{P}_{2} \mathbf{S}_{n+\alpha}+\frac{4}{9}\left[\left[S_{\mathbf{Y}}+K\left(\bar{E}_{n+\alpha}^{\mathrm{p}}\right)\right]^{2} K^{\prime}\left(\bar{E}_{n+\alpha}^{\mathrm{p}}\right) /\left(1-\frac{2}{3} \alpha \lambda_{n+\alpha} K^{\prime}\left(\bar{E}_{n+\alpha}^{\mathrm{p}}\right)\right)\right]}
$$

where

$$
\hat{\mathbf{C}}=\left(\mathbf{I}+\alpha \lambda_{n+\alpha} \mathbf{C} \mathbf{P}_{2}\right)^{-1} \mathbf{C}=\left(\mathbf{C}^{-1}+\alpha \lambda_{n+\alpha} \mathbf{P}_{2}\right)^{-1}
$$

represents the algorithmic tangent modulus. The symmetry of $\overline{\mathbf{C}}_{n+1}$ is evident.

\section{FIRST APPROXIMATION OF THE SHELL THEORY: MATRIX FORMULATION}

\subsection{First approximation of the shell theory}

Within the single director shell model, the following approximations of the above-presented geometrically non-linear shell theory may be introduced:

(i) It may be assumed that the components $E_{\alpha \beta}^{(2)}$ of the strain tensor are so small that they can be neglected, i.e. $E_{\alpha \beta}^{(2)} \rightarrow 0$. This is equivalent to $E_{i j}^{(2)} \rightarrow 0$, so the last part in the expression for the internal virtual work in (52) vanishes. This assumption leads to the (classical) linear distribution of the strain components $E_{\alpha \beta}$ through the shell thickness. Considering equation (40), it can be seen that in that case the relation $\mathbf{t}_{, \alpha} \cdot \mathbf{t}_{\beta} \approx \mathbf{T}_{, \alpha} \cdot \mathbf{T}_{\beta}$ is required, which holds for $\mathbf{R}_{, \alpha} \approx 0$ (see (17)). Then $\left.\mathbf{t}_{, \alpha} \cdot \mathbf{t}_{, \beta}=(\mathbf{R T})_{, \alpha} \cdot(\mathbf{R T})_{, \beta} \approx(\mathbf{R T})_{, \alpha}\right) \cdot\left(\mathbf{R T} \mathbf{T}_{, \beta}\right)=\mathbf{T}_{, \alpha} \cdot \mathbf{T}_{, \beta}$. The upper assumption therefore restricts the gradients of the tensor field $\mathbf{R}$ to be small. The order of the relative error for this approximation is given in Reference 9.

(ii) The variation of metric through the shell thickness may be neglected, i.e. $G_{i j} \approx A_{i j}$, $G^{i j} \approx A^{i j}$. Thus $\bar{\mu} \approx 1, \mathbf{Z} \approx \mathbf{I}$. This simplifies the constitutive relations in (60a) since $\mathrm{C}_{1}^{i j k l}$ and $\mathrm{C}_{3}^{i j k l}$ become zero.

\subsection{Matrix formulation}

In advance to the finite element formulation of the above approximate thin-shell model the kinematic and force variables are expressed in matrix notation. The matrix notation for vectorvalued quantities such as displacements and rotations is obvious. The strain tensor in matrix notation takes the form

$$
\boldsymbol{\varepsilon}_{n+1}=\left\{\begin{array}{c}
E_{11}^{(0)} \\
E_{22}^{(0)} \\
2 E_{12}^{(0)}
\end{array}\right\}_{n+1}, \quad \gamma_{n+1}=\left\{\begin{array}{l}
2 E_{13}^{(0)} \\
2 E_{23}^{(0)}
\end{array}\right\}_{n+1}, \quad \boldsymbol{\kappa}_{n+1}=\left\{\begin{array}{c}
E_{11}^{(1)} \\
E_{22}^{(1)} \\
2 E_{12}^{(1)}
\end{array}\right\}_{n+1}
$$

while the stress resultants (49)-(51) are written as

$$
\mathbf{n}_{n+1}=\left\{\begin{array}{l}
n^{11} \\
n^{22} \\
n^{12}
\end{array}\right\}_{n+1}, \quad \mathbf{q}_{n+1}=\left\{\begin{array}{l}
q^{13} \\
q^{23}
\end{array}\right\}_{n+1}, \quad \mathbf{m}_{n+1}=\left\{\begin{array}{l}
m^{11(1)} \\
m^{22(1)} \\
m^{12(1)}
\end{array}\right\}_{n+1}
$$


By employing the definitions of the second Piola-Kirchhoff stress resultants (49) and (50), the stress resultants (83) may be expressed in the familiar form as

$$
n_{n+1}^{\alpha \beta}=\int_{h^{-}}^{h^{+}} S_{n+1}^{\alpha \beta} \bar{\mu} \mathrm{d} \xi, \quad q_{n+1}^{\alpha 3}=\int_{h^{-}}^{h^{+}} S_{n+1}^{\alpha 3} \bar{\mu} \mathrm{d} \xi, \quad m_{n+1}^{\alpha \beta(1)}=\int_{h^{-}}^{h^{+}} S_{n+1}^{\alpha \beta} \xi \bar{\mu} \mathrm{d} \xi
$$

Following standard practice, the generalized strain-displacement operators are introduced in the matrix form as

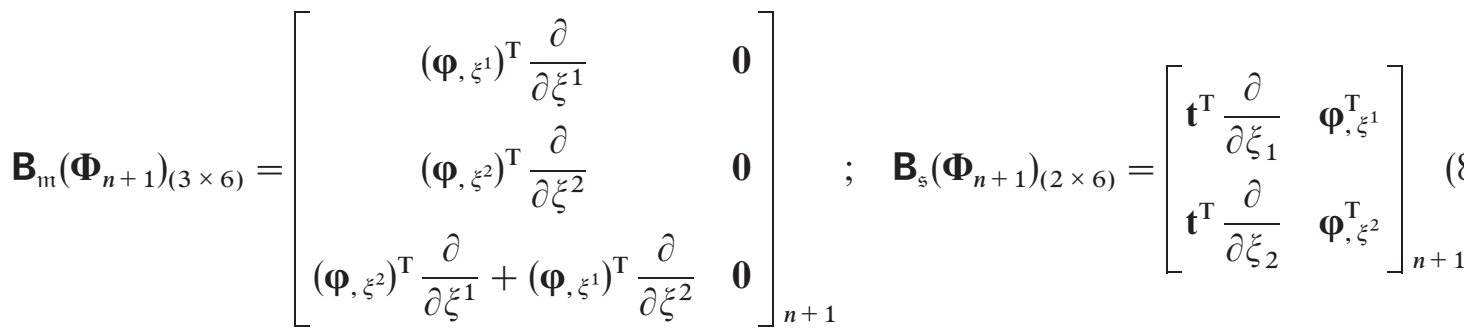

$$
\begin{aligned}
& \mathbf{B}_{\mathbf{b}}\left(\boldsymbol{\Phi}_{n+1}\right)_{(3 \times 6)}=\left[\begin{array}{cc}
\left(\mathbf{t}_{, \xi^{1}}\right)^{\mathrm{T}} \frac{\partial}{\partial \xi^{1}} & \left(\boldsymbol{\varphi}_{, \xi^{1}}\right)^{\mathrm{T}} \frac{\partial}{\partial \xi^{1}} \\
\left(\mathbf{t}_{, \xi^{2}}\right)^{\mathrm{T}} \frac{\partial}{\partial \xi^{2}} & \left(\boldsymbol{\varphi}_{, \xi^{2}}\right)^{\mathrm{T}} \frac{\partial}{\partial \xi^{2}} \\
\left(\mathbf{t}_{, \xi^{1}}\right)^{\mathrm{T}} \frac{\partial}{\partial \xi^{2}}+\left(\mathbf{t}_{, \xi^{2}}\right)^{\mathrm{T}} \frac{\partial}{\partial \xi^{1}} & \left(\boldsymbol{\varphi}_{, \xi^{1}}\right)^{\mathrm{T}} \frac{\partial}{\partial \xi^{2}}+\left(\boldsymbol{\varphi}_{, \xi^{2}}\right)^{\mathrm{T}} \frac{\partial}{\partial \xi^{1}}
\end{array}\right]_{n+1}
\end{aligned}
$$

which, in turn, define the membrane, shear and bending strain variations as

$$
\delta \boldsymbol{\varepsilon}=\mathbf{B}_{\mathfrak{m}}\left(\boldsymbol{\Phi}_{n+1}\right) \delta \boldsymbol{\Phi}, \quad \delta \gamma=\mathbf{B}_{\mathfrak{s}}\left(\boldsymbol{\Phi}_{n+1}\right) \delta \boldsymbol{\Phi}, \quad \delta \boldsymbol{\kappa}=\mathbf{B}_{\mathfrak{b}}\left(\boldsymbol{\Phi}_{n+1}\right) \delta \boldsymbol{\Phi}
$$

where the following compact notation is used:

$$
\delta \boldsymbol{\Phi}=\left\{\begin{array}{l}
\delta \mathbf{u} \\
\delta \mathbf{t}
\end{array}\right\}
$$

and $\delta \mathbf{t}$ is defined by relation (see (32) and (33))

$$
\delta \mathbf{t}=\boldsymbol{\Lambda}\left(\mathbf{s}_{n+1}\right)\left\{\begin{array}{l}
\delta \hat{s}^{1} \\
\delta \hat{s}^{2}
\end{array}\right\}
$$

where $\Lambda\left(\mathbf{s}_{n+1}\right)$ is a $(3 \times 2)$ matrix. It is sometimes convenient to express the relation (86a) in a compact form as

$$
\left\{\begin{array}{c}
\delta \boldsymbol{\varepsilon} \\
\delta \boldsymbol{\kappa} \\
\delta \gamma
\end{array}\right\}=\mathbf{B}\left(\boldsymbol{\Phi}_{n+1}\right) \delta \boldsymbol{\Phi}, \quad \mathbf{B}\left(\boldsymbol{\Phi}_{n+1}\right)_{(8 \times 6)}=\left[\begin{array}{l}
\mathbf{B}_{\mathfrak{m}} \\
\mathbf{B}_{\mathfrak{b}} \\
\mathbf{B}_{\mathfrak{s}}
\end{array}\right]_{n+1}
$$

where $\mathbf{B}\left(\boldsymbol{\Phi}_{n+1}\right)$ is the total strain-displacement matrix. 
The two-dimensional expression for the principle of the virtual work, (52) can now be written (with the assumptions (i) and (ii)) as

$$
\begin{aligned}
G\left(\mathbf{n}_{n+1}, \mathbf{m}_{n+1}, \mathbf{q}_{n+1}, \boldsymbol{\Phi}_{n+1} ; \delta \boldsymbol{\Phi}\right)= & \int_{\mathscr{M}}\left[\mathbf{n}_{n+1} \cdot \mathbf{B}_{\mathfrak{m}}\left(\boldsymbol{\Phi}_{n+1}\right) \delta \boldsymbol{\Phi}\right. \\
& \left.+\mathbf{m}_{n+1} \cdot \mathbf{B}_{\mathfrak{b}}\left(\boldsymbol{\Phi}_{n+1}\right) \delta \boldsymbol{\Phi}+\mathbf{q}_{n+1} \cdot \mathbf{B}_{\mathfrak{s}}\left(\boldsymbol{\Phi}_{n+1}\right) \delta \boldsymbol{\Phi}\right] \mathrm{d} A \\
& -G_{\text {ext }}(\delta \boldsymbol{\Phi})=0
\end{aligned}
$$

\section{FINITE ELEMENT FORMULATION}

In this section the finite element formulation is presented based on the total Lagrangian formulation and a quadrilateral shell element. Pure displacement formulations that arise from the direct finite element approximation of the weak form of equilibrium (87), result in poor performances caused by the shear locking behaviour in a thin-shell limit. It is therefore essential that solutions are based on some form of mixed formulation. Among various mixed formulations, hybrid-mixed methods seem to be well-suited for this class of problems (due to the strain-driven character of plasticity, assumed strain methods are more attractive than assumed stress methods). The four-node shell elements, based on displacement formulations, are not strongly affected by the membrane locking (the effect that is associated with the falsely activated membrane energy in bending-dominated problems). However, they exhibit very strong locking associated with the transverse shear strains. Therefore, approximation of the transverse shear strain field is performed by employing the so-called Assumed Natural Strain (ANS) concept, in the form first suggested by Dvorkin and Bathe. ${ }^{14}$

\subsection{Interpolation of the shell geometry}

As discussed in Section 2, the shell reference configuration can be completely described by the middle surface $\mathscr{M}$, the initial thickness $h_{0}$ and the unit vector field $\mathbf{T}$ normal to the middle surface $\mathscr{M}$. The adopted spatial finite element discretization relies on the isoparametric mapping from the parent element which is represented as the bi-unit square with co-ordinates $(\xi, \eta) \in[-1,1] \times[-1,1] \subset \mathbb{R}^{2}$. In the standard manner, the shell middle surface placement $\overline{\mathbf{X}} \in \mathscr{M}$ is defined as

$$
\bar{X}^{k}(\xi, \eta)=\sum_{I=1}^{4} N^{I}(\xi, \eta) \bar{X}_{I}^{k}, \quad(k=1,2,3)
$$

where $\bar{X}_{I}^{k}$ are the element nodal co-ordinates, while $N^{I}(\xi, \eta)$ are standard bi-linear shape functions

$$
N^{I}(\xi, \eta)=\frac{1}{4}\left(1+\xi \xi_{I}\right)\left(1+\eta \eta_{I}\right)
$$

with $\left(\xi_{I}, \eta_{I}\right) \in\{(-1,-1) ;(1,-1) ;(1,1) ;(-1,1)\}$ (see Figure 4).

In the approximation of the director field over the element, special consideration must be given to possible discretization errors, arising from the definition of the directors at the nodes of the finite element mesh. It seems that for shell elements with five degrees of freedom per node the most appropriate way to evaluate nodal directors is by averaging the nodal normals of the adjoined elements. ${ }^{5}$ The interpolation of the director field then may be given as

$$
T^{k}(\xi, \eta)=\sum_{I=1}^{4} N^{I}(\xi, \eta) T_{I}^{k}, \quad(k=1,2,3)
$$




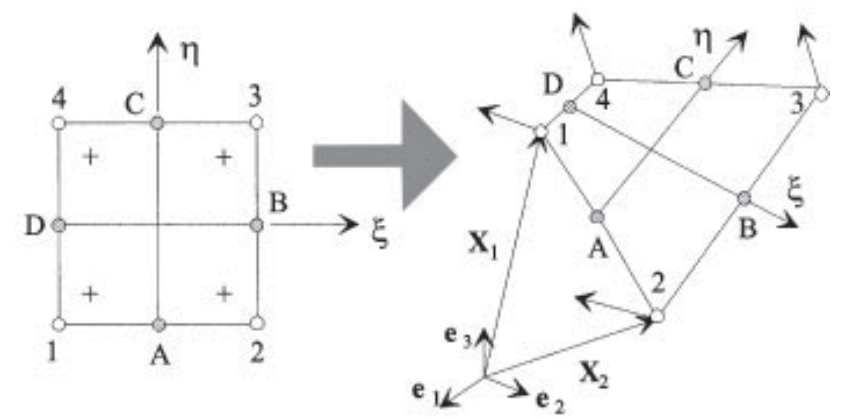

Figure 4. The four-node shell finite element

\subsection{Interpolation of physical fields}

The isoparametric discretization of the shell body displacement field described by (4) and (5) leads to

$$
\mathbf{U}=\sum_{I=1}^{4} N^{I}(\xi, \eta) \mathbf{U}_{I}=\sum_{I=1}^{4} N^{I}(\xi, \eta)(\mathbf{u}+\xi(\mathbf{t}-\mathbf{T}))_{I}
$$

Clearly, the middle surface displacement field is interpolated in the same way as the components of middle surface position vector.

The transverse shear field interpolation, based on the ANS method, prescribes the transverse shear to vary linearly between two opposite element edges (Figure 4). In the middle of each edge the shear is calculated with respect to the edge direction

$$
2 \bar{E}_{13}^{(0)}=\frac{1}{2}(1-\eta) 2 E_{13}^{(0) A}+\frac{1}{2}(1+\eta) 2 E_{13}^{(0) C}, \quad 2 \bar{E}_{23}^{(0)}=\frac{1}{2}(1-\xi) 2 E_{23}^{(0) D}+\frac{1}{2}(1+\xi) 2 E_{23}^{(0) B}
$$

where the middle surface points $\overline{\mathbf{X}}^{L}, L=A, B, C, D$ are defined with

$$
\overline{\mathbf{X}}^{L}=\frac{1}{2}\left(\overline{\mathbf{X}}\left(\xi_{M}, \eta_{M}\right)+\overline{\mathbf{X}}\left(\xi_{N}, \eta_{N}\right)\right)
$$

with $(L, M, N) \in\{(A, 1,2) ;(B, 2,3) ;(C, 3,4) ;(D, 1,4)\}$.

\subsection{Variations and derivatives of the physical fields}

In the discrete form of the virtual work expression for shells (87), the following variations and derivatives of the physical fields will be required: $\mathbf{u}_{, \zeta}, \delta \mathbf{u}_{, \zeta}, \delta \mathbf{t}, \mathbf{t}_{, \zeta}, \delta \mathbf{t}_{, \zeta}$, for $\zeta=\xi, \eta$ and $(\cdots)_{, \zeta}=\partial(\cdots) / \zeta$. Mid-surface displacement field variations are easily obtained as

$$
\mathbf{u}_{, \zeta}=\sum_{I=1}^{4} N_{, \zeta}^{I} \mathbf{u}_{I}, \quad \delta \mathbf{u}_{, \zeta}=\sum_{I=1}^{4} N_{, \zeta}^{I} \delta \mathbf{u}_{I}
$$

where $N_{, \zeta}^{I}$ denotes a partial derivative $\partial N^{I} / \zeta$. The rotational fields, according to (91), may be expressed as

$$
\mathbf{t}_{, \zeta}=\sum_{I=1}^{4} N_{, \zeta}^{I} \mathbf{t}_{I}, \quad \delta \mathbf{t}=\sum_{I=1}^{4} N^{I} \delta \mathbf{t}_{I}, \quad \delta \mathbf{t}_{, \zeta}=\sum_{I=1}^{4} N_{, \zeta}^{I} \delta \mathbf{t}_{I}
$$


where $\mathbf{t}_{I}$ is obtained by (23) and $\delta \mathbf{t}_{I}$ is given by (32) and (33). Thus, during the computational process it is necessary to construct local basis $\left\{\mathbf{E}_{i}\right\}$ at each nodal point. To this end definitions of $\mathbf{E}_{1}$ and $\mathbf{E}_{2}$ are usually required, since $\mathbf{E}_{3}=\mathbf{T}$ is already prescribed.

Linearization process further demands the second derivatives $\Delta(\delta \mathbf{t})$ and $\Delta\left(\delta \mathbf{t}_{, \zeta}\right)$ which may be obtained as

$$
\Delta(\delta \mathbf{t})=\sum_{I=1}^{4} N^{I} \Delta\left(\delta \mathbf{t}_{I}\right), \quad \Delta\left(\delta \mathbf{t}_{, \zeta}\right)=\sum_{I=1}^{4} N_{, \zeta}^{I} \Delta\left(\delta \mathbf{t}_{I}\right)
$$

\subsection{Discretized weak form of equilibrium}

The discretized weak form of the equilibrium equations (87) takes the form

$$
\begin{aligned}
G\left(\mathbf{n}_{n+1}, \mathbf{m}_{n+1}, \mathbf{q}_{n+1}, \boldsymbol{\Phi}_{n+1} ; \delta \boldsymbol{\Phi}\right)=\bigcup_{e=1}^{n_{\mathrm{el}}} \int_{\mathscr{A}_{e}}\left[\mathbf{n}_{n+1} \cdot \mathbf{B}_{\mathfrak{n}}\left(\boldsymbol{\Phi}_{n+1}\right) \delta \boldsymbol{\Phi}+\mathbf{m}_{n+1} \cdot \mathbf{B}_{\mathrm{mt}}\left(\boldsymbol{\Phi}_{n+1}\right) \delta \boldsymbol{\Phi}\right. \\
\left.+\mathbf{q}_{n+1} \cdot \mathbf{B}_{\mathfrak{s}}\left(\boldsymbol{\Phi}_{n+1}\right) \delta \boldsymbol{\Phi}\right] \mathrm{d} A-G_{\text {ext }}(\delta \boldsymbol{\Phi})=0
\end{aligned}
$$

where $\mathrm{d} A=\sqrt{A} \mathrm{~d} \xi \mathrm{d} \eta=\left\|\overline{\mathbf{X}}_{, \xi} \times \overline{\mathbf{X}}_{, \eta}\right\| \mathrm{d} \xi \mathrm{d} \eta$ and an integration domain $\mathscr{A}_{e}$ is a bi-unit square.

Since relations (92) are used to interpolate the transverse shear strain and stress resultants are given by (84), the above discretized weak form may be rewritten as

$$
\begin{aligned}
& G\left(\boldsymbol{\Phi}_{n+1} ; \delta \boldsymbol{\Phi}\right)=\bigcup_{e=1}^{n_{\mathrm{el}}} \int_{\mathscr{A}_{e}}\left[\mathbf{n}\left(\boldsymbol{\Phi}_{n+1}\right) \cdot \mathbf{B}_{\mathrm{n}}\left(\boldsymbol{\Phi}_{n+1}\right) \delta \boldsymbol{\Phi}+\mathbf{m}\left(\boldsymbol{\Phi}_{n+1}\right) \cdot \mathbf{B}_{\mathrm{mt}}\left(\boldsymbol{\Phi}_{n+1}\right) \delta \boldsymbol{\Phi}\right. \\
&\left.+\mathbf{q}\left(\boldsymbol{\Phi}_{n+1}\right) \cdot \overline{\mathbf{B}}_{\mathfrak{s}}\left(\boldsymbol{\Phi}_{n+1}\right) \delta \boldsymbol{\Phi}\right] \mathrm{d} A-G_{\mathrm{ext}}(\delta \boldsymbol{\Phi})=0
\end{aligned}
$$

where $\overline{\mathbf{B}}_{\mathfrak{s}}\left(\boldsymbol{\Phi}_{n+1}\right) \delta \boldsymbol{\Phi}$ is obtained by employing the interpolation (92) (see Reference 13 for details of implementation).

Remark 4. In order to simplify the elasto-plastic constitutive equations the local Cartesian frames are defined at numerical integration points. Details of implementation of this procedure are provided in Reference 13.

\section{SOLUTION PROCEDURE: LINEARIZATIONS ASPECTS}

The discrete form of the weak form of equilibrium for the present shell model given by (98) represents a set of highly non-linear equations with kinematic variables (nodal displacements and 'rotations') as unknowns. Usually, this problem is solved by a form of the Newton-Raphson iterative solution procedure. As an essential step of this procedure the linearization of this equation is required. In this section, the basic steps of this process are described. It should be observed that the linearization here is performed after the finite element discretization.

\subsection{Second derivatives of kinematic variables}

The second derivative of the $\boldsymbol{\Phi}=(\boldsymbol{\varphi}, \mathbf{t})$ required within the Newton-Raphson iterative process may be obtained as

$$
\mathrm{D}(\delta \mathbf{u}, \delta \mathbf{t})[\Delta \mathbf{u}, \Delta \mathbf{t}]=\left.\frac{\mathrm{d}}{\mathrm{d} \varepsilon}\right|_{\varepsilon=0}\left(\delta \mathbf{u}_{\varepsilon}, \delta \mathbf{t}_{\varepsilon}\right)=:\left[\begin{array}{c}
\Delta(\delta \mathbf{u}) \\
\Delta(\delta \mathbf{t})
\end{array}\right]
$$

It may easily be checked that $\mathrm{D}(\delta \mathbf{u})[\Delta \mathbf{u}, \Delta \mathbf{t}]$ is zero. 
In contrast, the second directional derivative of $\mathbf{t}$ (in the direction of $\Delta \mathbf{s}$ ) may be obtained as

$$
\Delta(\delta \mathbf{t})=: \mathrm{D}(\delta \mathbf{t})[\Delta \mathbf{s}]=\left.\frac{\mathrm{d}}{\mathrm{d} \varepsilon}\right|_{\varepsilon=0}(\delta \mathbf{t})_{\varepsilon}=\left.\frac{\mathrm{d}}{\mathrm{d} \varepsilon}\right|_{\varepsilon=0}[\delta \mathbf{t}(\mathbf{s}+\varepsilon \Delta \mathbf{s} ; \delta \mathbf{s})]
$$

The above expression is explicitly given as

$$
\begin{aligned}
\Delta(\delta \mathbf{t})= & \frac{\|\mathbf{s}\| \cos (\|\mathbf{s}\|)-\sin (\|\mathbf{s}\|)}{\|\mathbf{s}\|^{3}}[-(\delta \mathbf{s} \cdot \mathbf{s})(\mathbf{s} \cdot \Delta \mathbf{s}) \mathbf{T}+(\delta \mathbf{s} \cdot \Delta \mathbf{s}) \mathbf{s} \times \mathbf{T}] \\
& -\frac{\sin (\|\mathbf{s}\|)}{\|\mathbf{s}\|}(\delta \mathbf{s} \cdot \Delta \mathbf{s}) \mathbf{T}+\frac{\sin (\|\mathbf{s}\|)\left(3-\|\mathbf{s}\|^{2}\right)-3\|\mathbf{s}\| \cos (\|\mathbf{s}\|)}{\|\mathbf{s}\|^{5}}(\delta \mathbf{s} \cdot \mathbf{s})(\mathbf{s} \cdot \Delta \mathbf{s}) \mathbf{s} \times \mathbf{T} \\
& +\frac{\|\mathbf{s}\| \cos (\|\mathbf{s}\|)-\sin (\|\mathbf{s}\|)}{\|\mathbf{s}\|^{3}}[(\mathbf{s} \cdot \Delta \mathbf{s}) \delta \mathbf{s} \times \mathbf{T}+(\mathbf{s} \cdot \delta \mathbf{s}) \Delta \mathbf{s} \times \mathbf{T}]
\end{aligned}
$$

Details of the procedure describing implementation of the second derivatives of kinematic variables are provided in Appendix I.

\subsection{Newton-Raphson method}

The present problem may be stated as follows: Find a configuration $\boldsymbol{\Phi}_{n+1}$, such that $G\left(\boldsymbol{\Phi}_{n+1} ; \delta \boldsymbol{\Phi}\right)=0$ for any $\delta \boldsymbol{\Phi}$. Since the solution of the above non-linear problem is pursued by employing the Newton-Raphson method a linearization of $G\left(\boldsymbol{\Phi}_{n+1} ; \delta \boldsymbol{\Phi}\right)$ is required. The Newton-Raphson procedure at the configuration $\boldsymbol{\Phi}_{n+1}=\hat{\boldsymbol{\Phi}}_{n+1}$ may be expressed in the form

$$
\mathrm{D} G\left(\hat{\boldsymbol{\Phi}}_{n+1}^{(k)} ; \delta \boldsymbol{\Phi}\right)\left[\Delta \boldsymbol{\Phi}^{(k)}\right]=-G\left(\hat{\boldsymbol{\Phi}}_{n+1}^{(k)} ; \delta \boldsymbol{\Phi}\right)
$$

where the left-hand side of (102) supplies the tangent stiffness operator while the right-hand side has a standard interpretation as the unbalanced (residual) forces.

\subsection{Linearization of the virtual work functional}

Assuming the conservative loading, the tangent stiffness operator can be obtained by the directional derivative of the 'internal virtual work' in the direction $\Delta \mathbf{\Phi}=(\Delta \mathbf{u}, \Delta \mathbf{t})$ of incremental displacements and incremental 'rotations' at the discrete (nodal) points. It may be conventionally split into material and geometric parts:

$$
\mathrm{D} G\left(\boldsymbol{\Phi}_{n+1} ; \delta \boldsymbol{\Phi}\right)[\Delta \boldsymbol{\Phi}]=\mathrm{D}_{\mathrm{M}} G\left(\boldsymbol{\Phi}_{n+1} ; \delta \boldsymbol{\Phi}\right)[\Delta \boldsymbol{\Phi}]+\mathrm{D}_{\mathrm{G}} G\left(\boldsymbol{\Phi}_{n+1} ; \delta \boldsymbol{\Phi}\right)[\Delta \boldsymbol{\Phi}]
$$

where

$$
\begin{array}{rl}
\mathrm{D}_{\mathrm{M}} & G\left(\boldsymbol{\Phi}_{n+1} ; \delta \boldsymbol{\Phi}\right)[\Delta \boldsymbol{\Phi}] \\
\quad & \bigcup_{e=1}^{n_{\mathrm{el}}} \int_{\mathscr{A}_{e}}\left(\mathrm{D} n_{n+1}^{\alpha \beta}[\Delta \boldsymbol{\Phi}] \delta E_{\alpha \beta}^{(0)}+\mathrm{D}_{n+1}^{\alpha \beta}[\Delta \boldsymbol{\Phi}] \delta E_{\alpha \beta}^{(1)}+\mathrm{D} q_{n+1}^{\alpha}[\Delta \boldsymbol{\Phi}] 2 \delta E_{\alpha 3}^{(0)}\right) \mathrm{d} A \\
\mathrm{D}_{\mathrm{G}} G & \left(\boldsymbol{\Phi}_{n+1} ; \delta \boldsymbol{\Phi}\right)[\Delta \boldsymbol{\Phi}] \\
\quad=\bigcup_{e=1}^{n_{\mathrm{el}}} \int_{\mathscr{A}_{e}}\left(n_{n+1}^{\alpha \beta} \mathrm{D}\left(\delta E_{\alpha \beta}^{(0)}\right)[\Delta \boldsymbol{\Phi}]+m_{n+1}^{\alpha \beta} \mathrm{D}\left(\delta E_{\alpha \beta}^{(1)}\right)[\Delta \boldsymbol{\Phi}]+q_{n+1}^{\alpha} \mathrm{D}\left(2 \delta E_{\alpha 3}^{(0)}\right)[\Delta \boldsymbol{\Phi}]\right) \mathrm{d} A
\end{array}
$$

are, respectively, the material and geometric stiffness operators. 


\subsection{Material stiffness operator}

The material stiffness operator arises from the linearization of the constitutive equations at the fixed geometry. A starting point for the evaluation of the material stiffness operator is the first approximation of the expression (48) for the three-dimensional weak form of equilibrium. By employing the matrix notation (82)-(86) and finite element formulation described in Section 8, the tangent stiffness matrix may then be written as

$$
\begin{aligned}
& \mathrm{D}_{\mathrm{M}} G\left(\boldsymbol{\Phi}_{n+1} ; \delta \boldsymbol{\Phi}\right)[\Delta \boldsymbol{\Phi}] \\
& \quad=\bigcup_{e=1}^{n_{\mathrm{cl}}} \int_{\mathscr{A}_{e}}\left(\mathbf{B}\left(\boldsymbol{\Phi}_{n+1}\right) \delta \boldsymbol{\Phi} \cdot\left[\begin{array}{ccc}
{\left[\overline{\mathbf{C}}_{n+1}\right]_{m 0}} & {\left[\overline{\mathbf{C}}_{n+1}\right]_{m 1}} & {\left[\overline{\mathbf{C}}_{n+1}\right]_{m s 0}} \\
{\left[\overline{\mathbf{C}}_{n+1}\right]_{m 1}} & {\left[\overline{\mathbf{C}}_{n+1}\right]_{m 2}} & {\left[\overline{\mathbf{C}}_{n+1}\right]_{m s 1}} \\
{\left[\overline{\mathbf{C}}_{n+1}\right]_{m s 0}} & {\left[\overline{\mathbf{C}}_{n+1}\right]_{m s}} & {\left[\overline{\mathbf{C}}_{n+1}\right]_{s 0}}
\end{array}\right] \mathbf{B}\left(\boldsymbol{\Phi}_{n+1}\right) \Delta \boldsymbol{\Phi}\right) \mathrm{d} A
\end{aligned}
$$

with the submatrices defined as

$$
\left[\overline{\mathbf{C}}_{n+1}\right]_{m J}=\int_{h^{-}}^{h^{+}} \overline{\mathbf{C}}_{m}(\xi)^{J} \bar{\mu} \mathrm{d} \xi, \quad\left[\overline{\mathbf{C}}_{n+1}\right]_{m s J}=\int_{h^{-}}^{h^{+}} \overline{\mathbf{C}}_{m s}(\xi)^{J} \bar{\mu} \mathrm{d} \xi, \quad\left[\overline{\mathbf{C}}_{n+1}\right]_{s 0}=\int_{h^{-}}^{h^{+}} \overline{\mathbf{C}}_{s} \bar{\mu} \mathrm{d} \xi
$$

where

$$
\left[\overline{\mathbf{C}}_{n+1}\right]=\left[\begin{array}{ll}
\overline{\mathbf{C}}_{m} & \overline{\mathbf{C}}_{m s} \\
\overline{\mathbf{C}}_{m s} & \overline{\mathbf{C}}_{s}
\end{array}\right]_{n+1}
$$

represents membrane, shear and combined membrane-shear parts of the consistent tangent stiffness matrix (81).

Remark 5. The material part of the tangent stiffness matrix and internal stress resultants are evaluated by numerical integration through the thickness of the shell. It is worth noting that the classical (Newton-Cotes or Gaussian) quadrature formulae are designed for the purpose of integrating continuous and smooth functions. However, in the present work, functions may neither be smooth nor continuous. The influence of this defect on the accuracy of numerical results is, in general, difficult to estimate and further research is required.

\subsection{Derivatives of strain measure variations}

Derivatives of strain measure variations $(\delta \boldsymbol{\varepsilon}, \delta \gamma, \delta \mathbf{\kappa})$ in the direction of $\Delta \mathbf{\Phi}=(\Delta \mathbf{u}, \Delta \mathbf{t})$ may be expressed as

$$
\begin{aligned}
\mathrm{D}\left(\delta E_{\alpha \beta}^{(0)}\right)[\Delta \mathbf{u}, \Delta \mathbf{t}]= & \frac{1}{2}\left(\delta \mathbf{u}_{, \alpha} \cdot \Delta\left(\mathbf{u}_{, \beta}\right)+\Delta\left(\mathbf{u}_{, \alpha}\right) \cdot \delta \mathbf{u}_{, \beta}\right) \\
\mathrm{D}\left(2 \delta E_{\alpha 3}^{(0)}\right)[\Delta \mathbf{u}, \Delta \mathbf{t}]= & \left(\Delta\left(\mathbf{u}_{, \alpha}\right) \cdot \delta \mathbf{t}+\delta \mathbf{u}_{, \alpha} \cdot \Delta \mathbf{t}+\boldsymbol{\varphi}_{n+1, \alpha} \cdot \mathrm{D}(\delta \mathbf{t})[\Delta \mathbf{t}]\right) \\
\mathrm{D}\left(\delta E_{\alpha \beta}^{(1)}\right)[\Delta \mathbf{u}, \Delta \mathbf{t}]= & \frac{1}{2}\left(\Delta\left(\mathbf{u}_{, \alpha}\right) \cdot \delta \mathbf{t}_{, \beta}+\Delta\left(\mathbf{u}_{, \beta}\right) \cdot \delta \mathbf{t}_{, \alpha}+\delta \mathbf{u}_{, \alpha} \cdot \Delta\left(\mathbf{t}_{, \beta}\right)+\delta \mathbf{u}_{, \beta} \cdot \Delta\left(\mathbf{t}_{, \alpha}\right)\right. \\
& \left.+\boldsymbol{\varphi}_{n+1, \alpha} \cdot \mathrm{D}\left(\delta \mathbf{t}_{, \beta}\right)[\Delta \mathbf{t}]+\boldsymbol{\varphi}_{n+1, \beta} \cdot \mathrm{D}\left(\delta \mathbf{t}_{, \alpha}\right)[\Delta \mathbf{t}]\right)
\end{aligned}
$$

\subsection{Geometric stiffness operator}

The geometric stiffness operator arises from the linearization of the geometric part, when holding the material part fixed. It is recovered from the second integral in (104) by employing the 
following relationships:

$$
\begin{aligned}
n_{n+1}^{\alpha \beta} \mathrm{D}\left(\delta E_{\alpha \beta}^{(0)}\right)[\Delta \mathbf{u}, \Delta \mathbf{t}]= & \sum_{I=1}^{4} \sum_{K=1}^{4} \delta \mathbf{u}_{I} \cdot \Delta \mathbf{u}_{K}\left(\frac{1}{2} n_{n+1}^{\alpha \beta}\left(N_{, \alpha}^{K} N_{, \beta}^{I}+N_{, \beta}^{K} N_{, \alpha}^{I}\right)\right) \\
m_{n+1}^{\alpha \beta} \mathrm{D}\left(\delta E_{\alpha \beta}^{(1)}\right)[\Delta \mathbf{u}, \Delta \mathbf{t}]= & \sum_{I=}^{4} \sum_{K=1}^{4} \delta \mathbf{t}_{I} \cdot \Delta \mathbf{u}_{K}\left(\frac{1}{2} m_{n+1}^{\alpha \beta}\left(N_{, \alpha}^{K} N_{, \beta}^{I}+N_{, \beta}^{K} N_{, \alpha}^{I}\right)\right) \\
& +\sum_{I=1}^{4} \sum_{K=1}^{4} \delta \mathbf{u}_{I} \cdot \Delta \mathbf{t}_{K}\left(\frac{1}{2} m_{n+1}^{\alpha \beta}\left(N_{, \alpha}^{I} N_{, \beta}^{K}+N_{, \beta}^{I} N_{, \alpha}^{K}\right)\right) \\
& +\sum_{I=1}^{4} \Delta\left(\delta \mathbf{t}_{I}\right) \cdot\left(\frac{1}{2} m_{n+1}^{\alpha \beta}\left(\boldsymbol{\varphi}_{n+1, \alpha} N_{, \beta}^{I}+\boldsymbol{\varphi}_{n+1, \beta} N_{, \alpha}^{I}\right)\right) \\
q_{n+1}^{\alpha} \mathrm{D}\left(2 \delta E_{\alpha 3}\right)[\Delta \mathbf{u}, \Delta \mathbf{t}]= & \sum_{I=}^{4} \sum_{K=1}^{4} \delta \mathbf{t}_{I} \cdot \Delta \mathbf{u}_{K}\left(q_{n+1}^{\alpha} N_{, \alpha}^{K} N^{I}\right) \\
& +\sum_{I=1}^{4} \sum_{K=1}^{4} \delta \mathbf{u}_{I} \cdot \Delta \mathbf{t}_{K}\left(q_{n+1}^{\alpha} N_{, \alpha}^{I} N^{K}\right) \\
& +\sum_{I=1}^{4} \Delta\left(\delta \mathbf{t}_{I}\right) \cdot\left(q_{n+1}^{\alpha} \boldsymbol{\varphi}_{n+1, \alpha} N^{I}\right)
\end{aligned}
$$

Linearization of the transverse shear part based on interpolation (92) is not considered here. Implementation details may be found in Reference 13 .

\section{NUMERICAL EXAMPLES}

In this section, a set of numerical examples is presented in order to illustrate the range of applications and performance capabilities of the described finite rotation elasto-plastic shell model. The non-linear equations are solved by the full Newton-Raphson method in all examples. The deformation paths are followed by carrying out either force control or by the cylindrical arc-length method as described in Reference 23. In all examples the local tolerance, when iterating for the incremental plastic multiplier, is set to $1.0 \times 10^{-8}$, while the global convergence is achieved when the Euclidean norm of the residual is less than $1.0 \times 10^{-6}$. Whenever it is possible comparisons wih results in the literature are presented.

Example 1. Failure analysis of the Scordelis-Lo roof. Geometry and material characteristics for this example are given in Figure 5. The half-length of the shell is $L=7.6 \mathrm{~m}$, radius is $R=7.6 \mathrm{~m}$ and thickness is $h_{0}=0.076 \mathrm{~m}$. The material parameters of the roof are: elastic modulus is $E=2 \cdot 1 \times 10^{4} \mathrm{~N} / \mathrm{mm}^{2}$, Poisson's ratio is $v=0 \cdot 0$, yield stress is $S_{\mathrm{Y}}=4 \cdot 2 \mathrm{~N} / \mathrm{mm}^{2}$ and hardening parameter is assumed to be equal to $H=0 \cdot 0$. The gravity-type loading of the shell is considered with the reference value of $f_{0}=4.0 \mathrm{kN} / \mathrm{m}^{2}$. Due to the symmetry conditions, the analysis is performed for one quarter of the roof, restricting deformations to be symmetric along the lines $X_{1}=0$ and $X_{2}=0$. The displacement boundary conditions can be, in relation to Figure 5, expressed as

$$
\begin{aligned}
& U_{1}=0, \quad \varphi_{2}=0 \quad \text { at } X_{1}=0 \\
& U_{2}=0, \quad \varphi_{1}=0 \quad \text { at } X_{2}=0 \\
& U_{1}=U_{3}=0, \quad \varphi_{2}=0 \quad \text { at } X_{2}=L
\end{aligned}
$$

where $U_{i}$ are displacements in $X_{i}$-directions and $\varphi_{\alpha}$ are rotations about the $X_{\alpha}$-axis. 


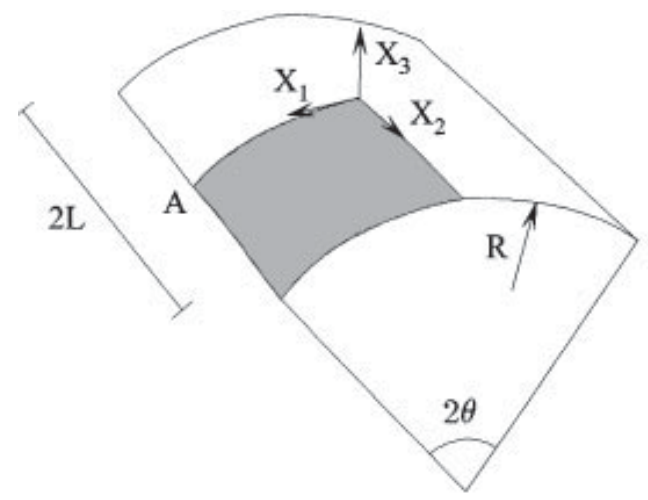

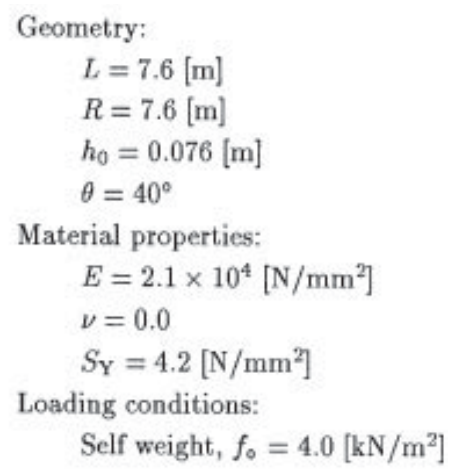

Figure 5. Scordelis-Lo roof: geometry, material characteristics and loading conditions

This example has been analysed by many authors with the most complete non-linear analyses completed by Perić and Owen ${ }^{24}$ and Crisfield and Peng. ${ }^{25}$ In both publications, the authors used the faceted shell formulations based on Morley thin-shell triangular elements, which do not account for the transverse shear strains.

Full finite deformation kinematics is considered in the analysis and the computation is performed employing standard arc-length control. ${ }^{23}$ Simpson's integration rule with seven equidistant integration points is adopted for the numerical integration across the shell thickness. Two different meshes of $32 \times 32$ and $50 \times 50$ four-node elements for one quarter of the roof are used to simulate elasto-plastic shell deformations.

Gravitational load versus vertical displacement curves for point A are plotted in Figure 6. Both meshes give virtually the same results for deformation prior to the maximum load, and compare well with the results of Peric and Owen and Crisfield and Peng, obtained with a mesh of $16 \times 16$ triangular Morley elements. This, however, cannot be said for the force-displacement curves in the section of non-stable equilibrium configurations. A sharp drop, observed in all diagrams, occurs at $U_{3} \approx-1.0 \mathrm{~m}$ in References 24 and 25 , while it is noticed at $U_{3} \approx-1.3 \mathrm{~m}$ in the present analysis. The differences in results may relate to the differences in the finite element formulations.

It is interesting to note that a different mode of failure is observed as well. The very localized failure mode of the roof can be evident from Figure 7, where five equilibrium configurations for the mesh $50 \times 50$ are plotted. The displacements of point $A$ at the configurations denoted by a, b, $\mathrm{c}$ and $\mathrm{d}$ in Figure 7 are also marked in Figure 6. The failure starts through formation of a plastic hinge about $X_{2}$ axis in the central part of the shell accompanied by the appearance of another elliptically shaped plastic hinge (see Figures 7(a) and 7(b)). Both hinges meet at two points, seen clearly in Figure 7(c). The formation of the plastic hinges in the central part of the shell is followed by the plastic hinge formation which leads from the two mentioned points towards the corners of the roof.

The Morley thin-shell element and the four-node bilinear shell element thus produce two different modes of failure (see Figure 5.8 in Reference 24 for comparison). A possible explanation may be that the Morley thin-shell element does not account for the transverse shear deformation. Also the higher geometric flexibility of the triangular-type shell element over the structured quadrilateral element mesh may be a reason for different behaviours in the representation of these highly localized modes of failure.

The Euclidean norm of residuals $\|\mathbf{G}\|$ for two typical load steps given in Table I clearly illustrate quadratic rate of asymptotic convergence typical for the Newton-Raphson-based 


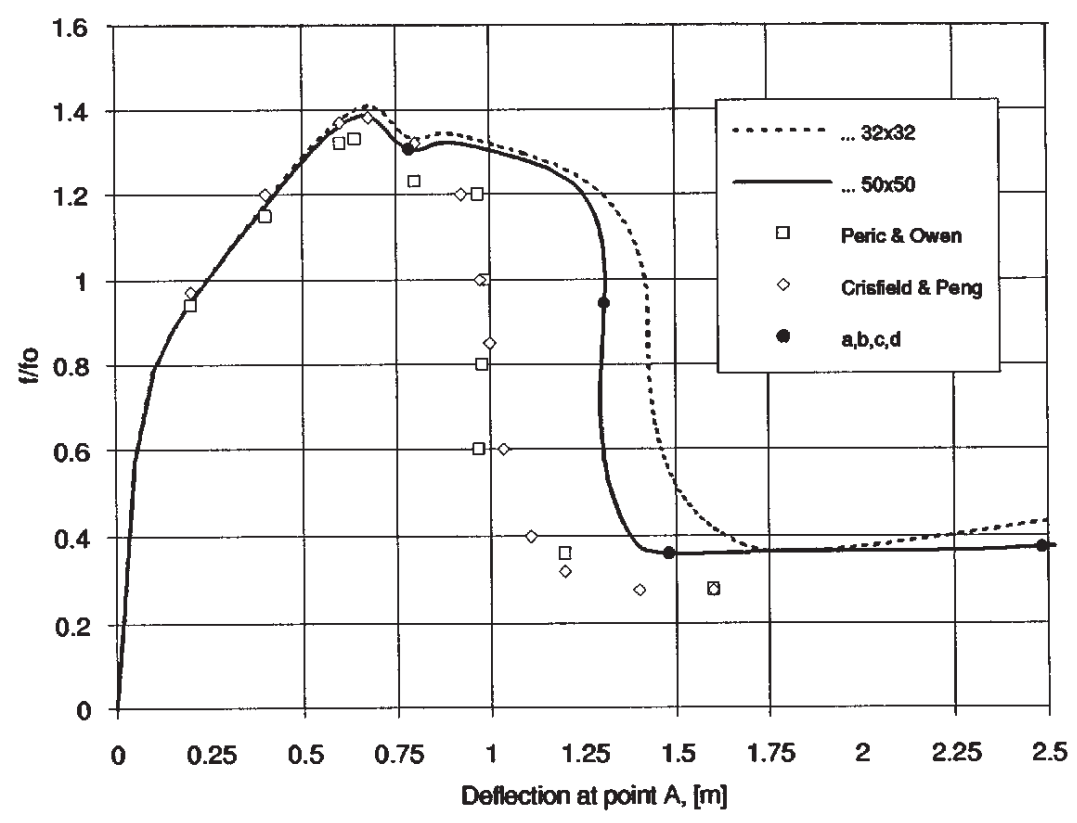

Figure 6. Scordelis-Lo roof: load versus displacement curves for point A

iterative procedure. The final configuration for the finer mesh, corresponding to the point A displacement of $U_{3}=-2.9385 \mathrm{~m}$, was attained in 150 load steps.

Example 2. Elasto-plastic buckling of cylindrical shell. Sobel and Newman ${ }^{26}$ performed experimental tests for the (axisymmetric) plastic buckling of axially compressed steel cylinders. The geometry characteristics of the typical specimen, denoted by $C-2$ in Reference 26 are: length is $L=127.1 \mathrm{~mm}$, thickness is $h_{0}=2.36 \mathrm{~mm}$ and radius is $R=43.33 \mathrm{~mm}$. The points of the stress-strain hardening curve of the steel considered are given in Table II and the Poisson ratio is given as $0 \cdot 274$. Thickness-to-radius ratio $\left(h_{0} / R \approx 0 \cdot 054\right)$ is chosen to be such that the buckling occurs in the plastic range (see Figure 8).

During the experimental testing procedure, all cylinders initially developed a short axisymmetric bulge of half sine wave near one or both ends. Upon further increasing the load, the bulge continued to grow and after the load had reached its maximum value the bulge developed into an axisymmetric ring. The maximum load the cylinder was able to sustain before the first ring occurred was defined as the buckling load. Its values for the three cylinders of length $L \approx 127.0$ are reported as $202 \cdot 83,212.17$ and $183.70 \mathrm{MPa}$, where the last value was not considered to be very accurate. It is interesting to note that the buckling load for the cylinder of length $L=254.3 \mathrm{~mm}$, which is twice the length of other specimens, is reported to be $216.6 \mathrm{MPa}$, which indicates that the ratio $h_{0} / L$ has small influence on the buckling load in this case. Additionally, Sobel and Newman numerically estimated the buckling load with their computer programme. They reported the value of $189.5 \mathrm{MPa}$, when the cylinder with the above given geometrical characteristics was simply supported at its ends.

In the present analysis, the mesh of $32 \times 48$ four-node elements is used (due to the symmetry conditions) to generate one octant of the same cylinder. Two cases, simply supported and clamped, are considered. The radial expansion of the cylinder ends is prevented in both cases, since due to the friction between the cylinder and the loading plates its ends cannot move freely. The solutions for the buckling load for the simply supported and clamped case were 186.3 and 


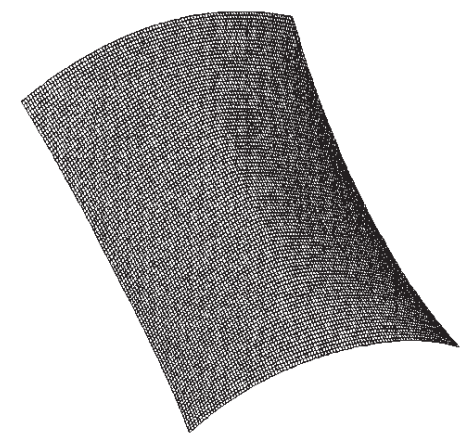

a
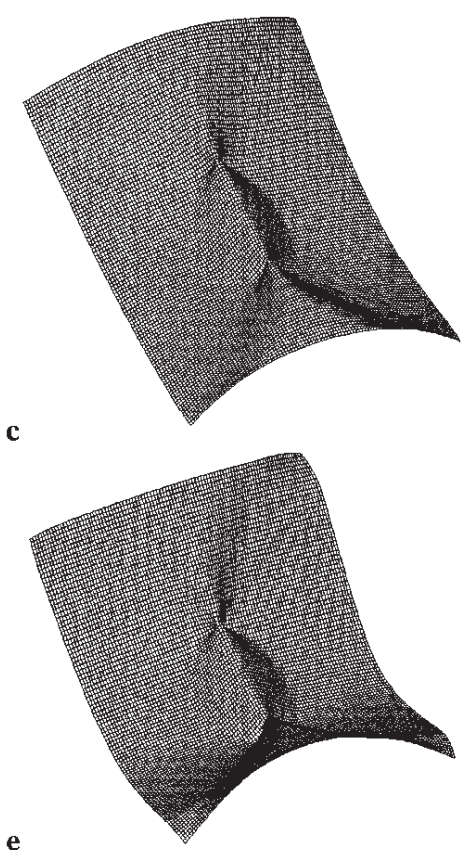

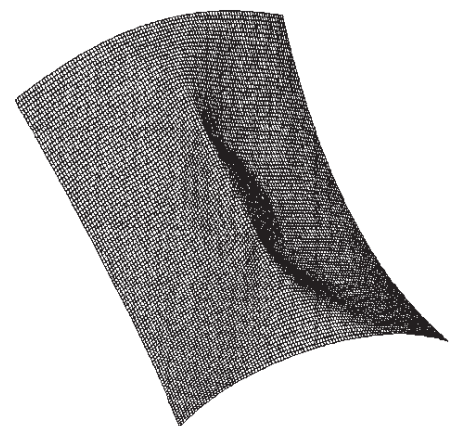

b
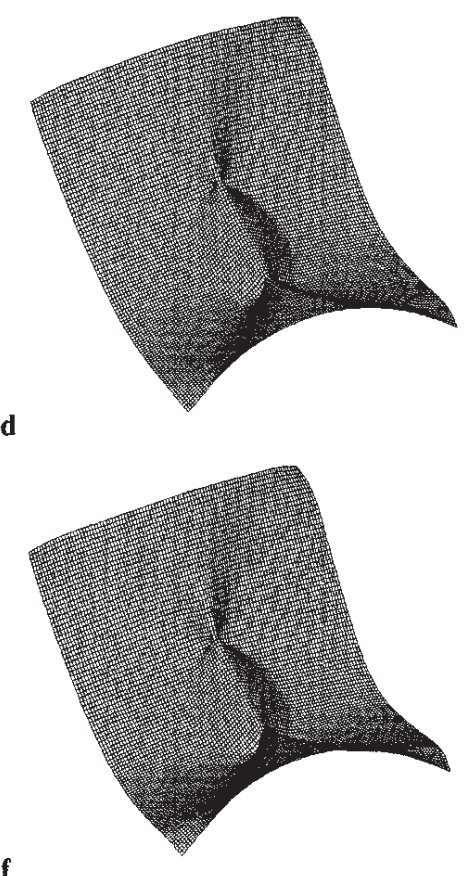

Figure 7. Scordelis-Lo roof: deformed configuration of the shell at five different load stages

Table I. Failure analysis of Scordelis-Lo roof: Euclidean norms of residuals for two typical load steps for the mesh $(32 \times 32)$

\begin{tabular}{lll}
\hline Iteration & $f / f_{0}=1.256$ & $f / f_{0}=1 \cdot 400$ \\
\hline 1 & $0 \cdot 1018 \times 10^{+3}$ & $0 \cdot 1367 \times 10^{+3}$ \\
2 & $0 \cdot 2399 \times 10^{+2}$ & $0 \cdot 6869 \times 10^{+2}$ \\
3 & $0 \cdot 3033 \times 10^{+1}$ & $0 \cdot 3996 \times 10^{+2}$ \\
4 & $0 \cdot 1971 \times 10^{0}$ & $0 \cdot 6924 \times 10^{+1}$ \\
5 & $0 \cdot 3120 \times 10^{-2}$ & $0 \cdot 2423 \times 10^{+1}$ \\
6 & $0 \cdot 1198 \times 10^{-6}$ & $0 \cdot 8672 \times 10^{0}$ \\
7 & & $0 \cdot 1634 \times 10^{0}$ \\
8 & & $0 \cdot 4515 \times 10^{-2}$ \\
9 & & $0 \cdot 1868 \times 10^{-6}$ \\
\hline
\end{tabular}


Table II. Elasto-plastic buckling of cylindrical shell: hardening curve

\begin{tabular}{ccc}
\hline Point & Stress $S\left(\mathrm{~N} / \mathrm{mm}^{2}\right)$ & Eq. plastic strain, $\bar{E}^{\mathrm{p}}(\%)$ \\
\hline 1 & $162 \cdot 722$ & $0 \cdot 000$ \\
2 & $231 \cdot 672$ & $0 \cdot 100$ \\
3 & $248 \cdot 910$ & $0 \cdot 255$ \\
4 & $268 \cdot 905$ & $0 \cdot 425$ \\
5 & $286 \cdot 143$ & $0 \cdot 675$ \\
6 & $295 \cdot 800$ & $0 \cdot 925$ \\
7 & $313 \cdot 723$ & $1 \cdot 425$ \\
8 & $329 \cdot 581$ & $2 \cdot 425$ \\
9 & $373 \cdot 709$ & $5 \cdot 425$ \\
10 & $428 \cdot 180$ & $9 \cdot 925$ \\
\hline
\end{tabular}

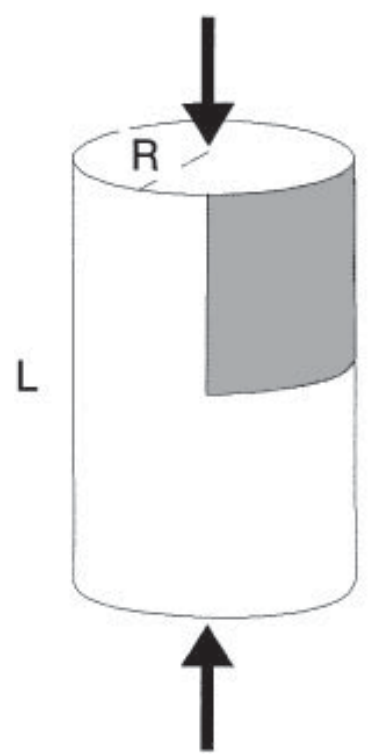

$$
\begin{aligned}
& \text { Geometry: } \\
& L=127.1[\mathrm{~mm}] \\
& R=43.33[\mathrm{~mm}] \\
& h_{0}=2.36[\mathrm{~mm}] \\
& \text { Material properties: } \\
& E=216,962\left[\mathrm{~N} / \mathrm{mm}^{2}\right] \\
& \nu=0.274 \\
& S_{\mathrm{Y}}=162.722\left[\mathrm{~N} / \mathrm{mm}^{2}\right]
\end{aligned}
$$

Figure 8. Cylindrical shell: geometry, material characteristics and loading conditions

$195 \cdot 1 \mathrm{kN}$, respectively. The clamped case gives higher values, which is in accordance with the numerical observations of Sobel and Newman. The axial force versus axial displacement curves are depicted in Figure 9 for both cases along with the experimentally obtained buckling loads for the three shorter cylinders. The buckling load obtained by Sobel and Newman is slightly higher than the buckling load obtained by the present analysis. Figures 10 and 11 show the finite element representation of development of the first axisymmetric ring for both simply supported and clamped cases.

Example 3. Pinched elasto-plastic cylinder with isotropic hardening. This example, pinching of a cylinder, was considered by Simo and Kennedy, ${ }^{27}$ who used the generalized Ilyushin-Shapiro elasto-plastic model, formulated entirely in stress resultants. The short cylinder, bounded by two rigid diaphragms at its ends is pinched by two concentrated forces at its middle section. The 


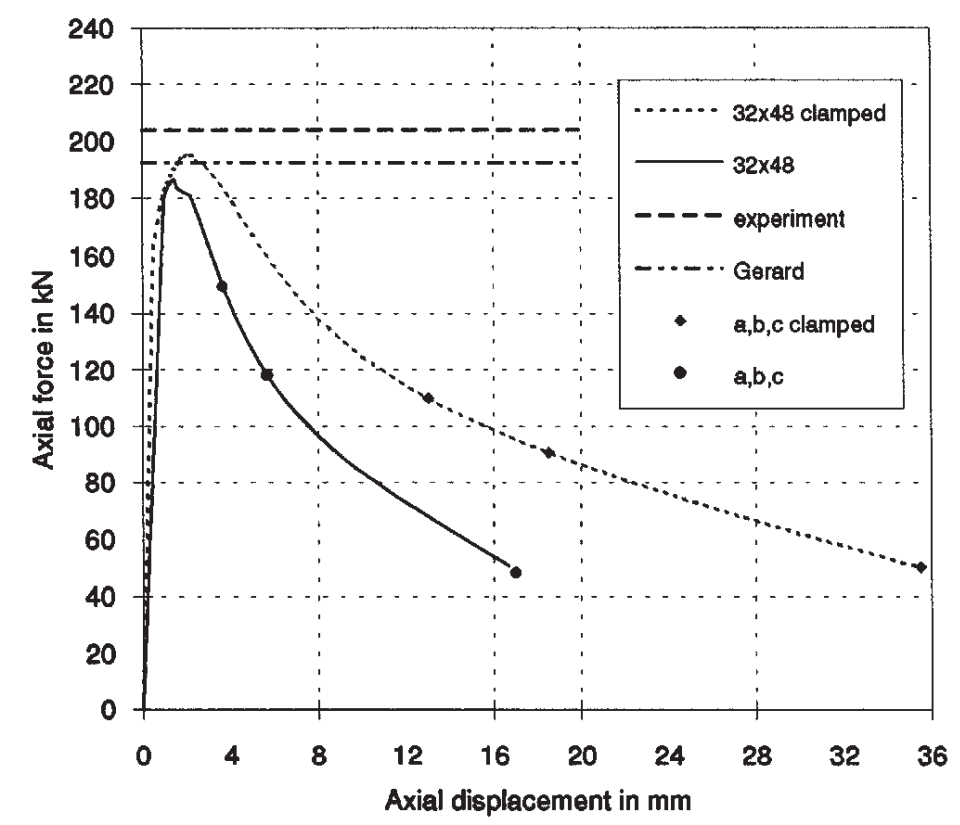

Figure 9. Cylindrical shell: axial force versus axial displacement curves

a

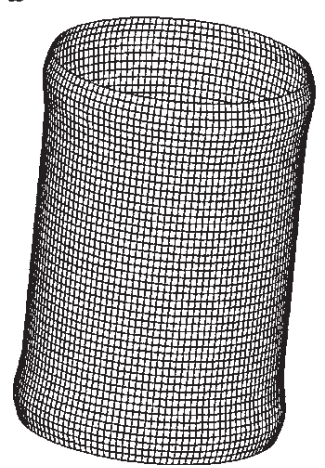

c

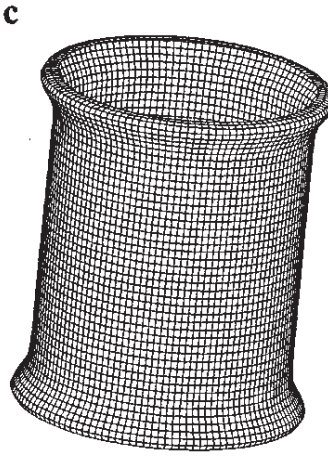

b

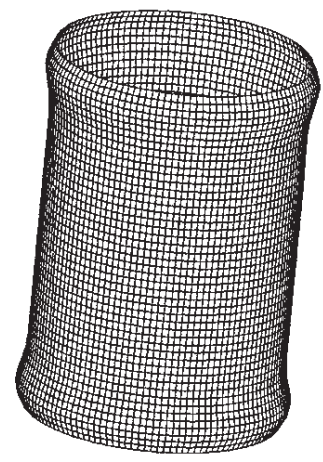

Figure 10. Simply supported cylindrical shell: deformed finite element mesh at various stages of loading

geometry is shown in Figure 12. Due to the symmetry, only one octant of the cylinder is modelled with a mesh of $32 \times 32$ four-node elements. The half-length of a cylinder is $L=300$, the radius is $R=300$ and the thickness is $h_{0}=3 \cdot 0$. The material is characterized by an isotropic hardening 

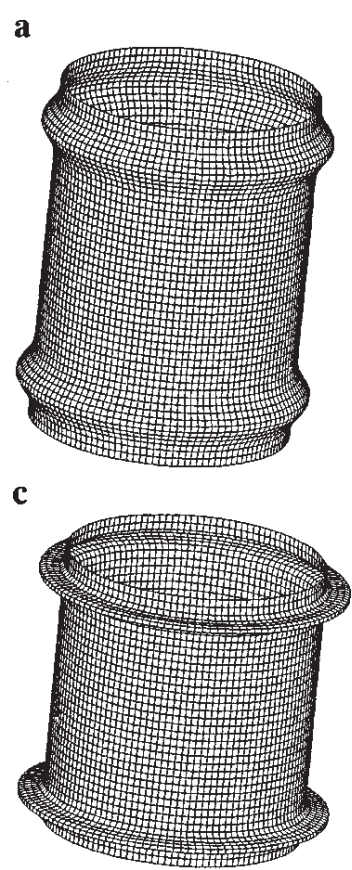

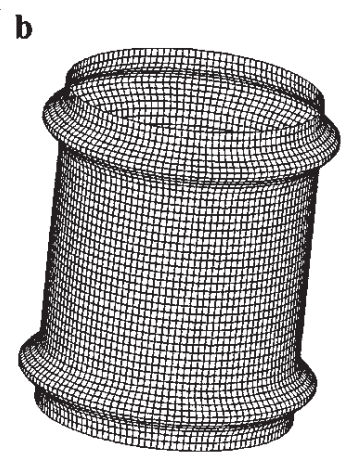

Figure 11. Clamped cylindrical shell: deformed finite element mesh at various stages of loading

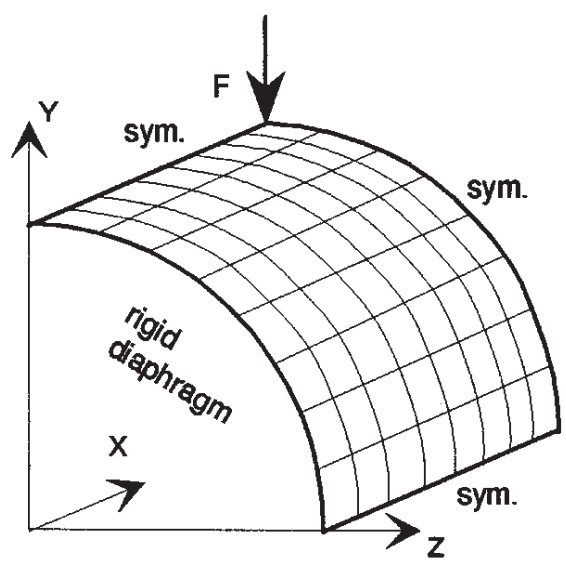

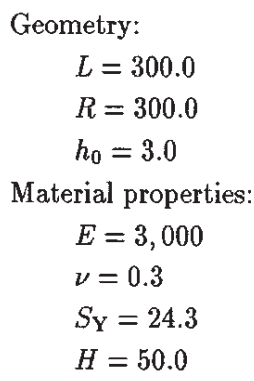

Loading conditions:

Point load, $F_{0}=1,000$

Figure 12. Pinched elasto-plastic cylinder: geometry, material characteristics and loading conditions

plastic response. The material properties are assumed to be: elastic modulus is $E=3000$, Poisson's ratio is $v=0 \cdot 3$, yield stress is $S_{\mathrm{Y}}=24 \cdot 3$ and hardening parameter is $H=50 \cdot 0$. All geometrical and material characteristics are equal to those in Reference 27, except the hardening parameter, since the hardening parameters of the shell model and the classical $J_{2}$ model cannot be related explicitly. As shown in Reference 27, the linear isotropic hardening response of the shell model is considerably different compared to the classical $J_{2}$ theory for the same value of the hardening parameter.

In the present approach, the numerical integration through the shell thickness was performed by Simpson's integration rule with seven integration points. In the Figure 13 the pinching load is 


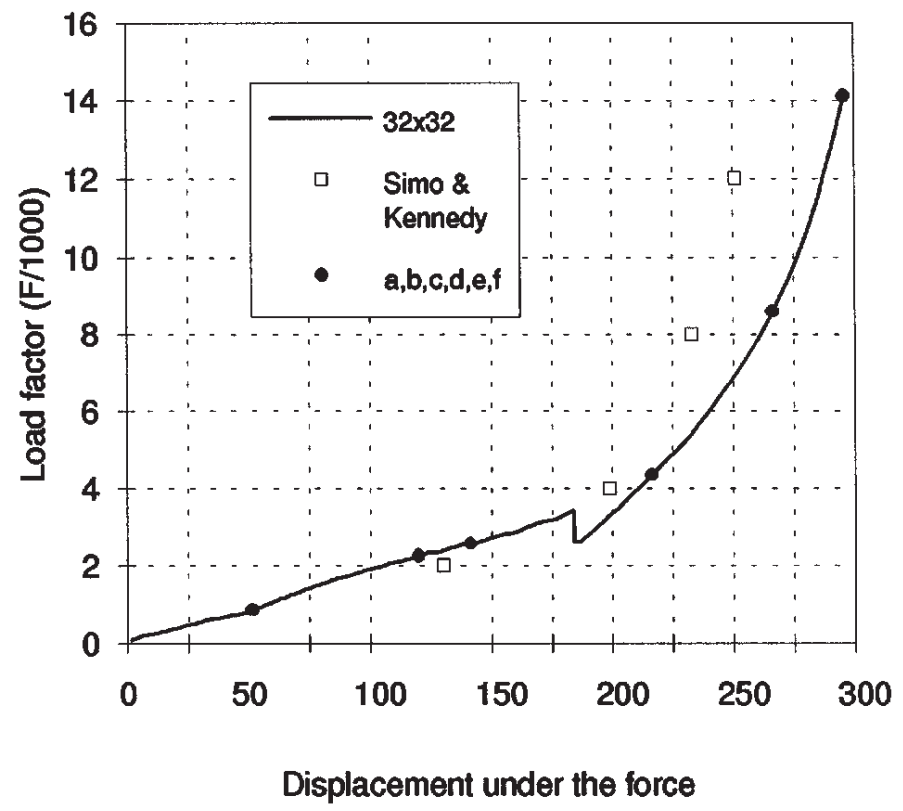

Figure 13. Pinched elasto-plastic cylinder: displacements under the force

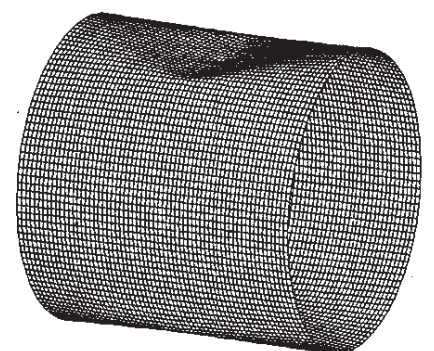

c

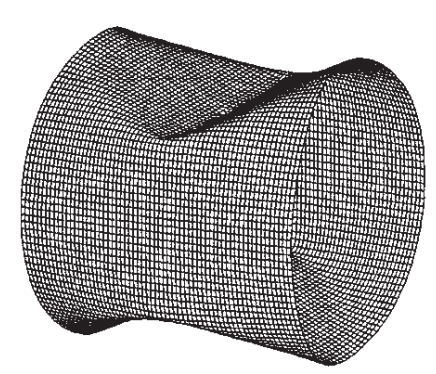

e

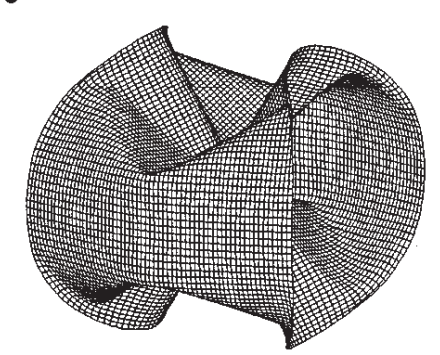

b

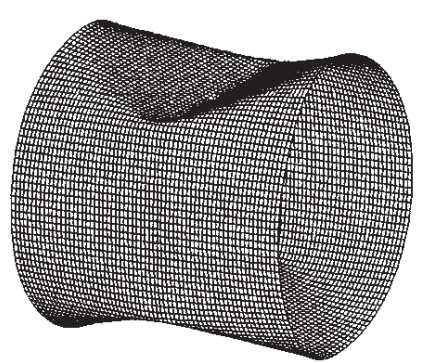

d

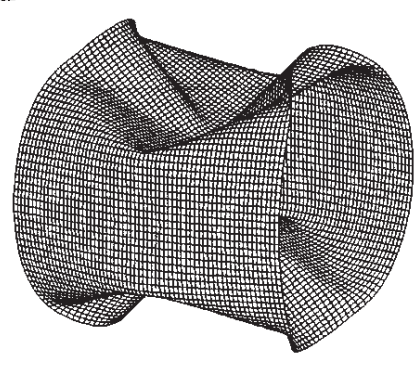

f

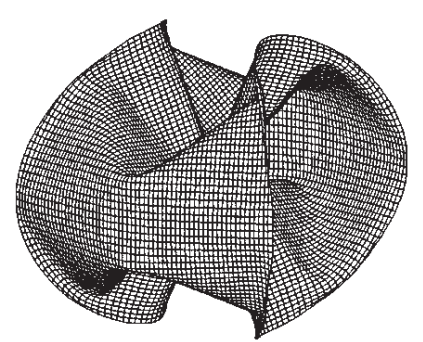

Figure 14. Pinched elasto-plastic cylinder: deformed finite element meshes for different load stages 
Table III. Pinched elasto-plastic cylinder with isotropic hardening: Euclidean norms of residuals and maximum norms of displacement/rotation for a load step $F=2 \cdot 6655 \times 10^{-3}$

\begin{tabular}{lll}
\hline Iteration & \multicolumn{1}{c}{$\| \mathbf{G}$} & \multicolumn{1}{c}{$\max |U|$} \\
\hline 1 & $0.5802 \times 10^{+4}$ & $0 \cdot 3455 \times 10^{+1}$ \\
2 & $0 \cdot 1352 \times 10^{+4}$ & $0 \cdot 4677 \times 10^{0}$ \\
3 & $0 \cdot 1196 \times 10^{+3}$ & $0 \cdot 1359 \times 10^{0}$ \\
4 & $0 \cdot 3919 \times 10^{+1}$ & $0 \cdot 2922 \times 10^{-1}$ \\
5 & $0 \cdot 3975 \times 10^{0}$ & $0 \cdot 1204 \times 10^{-2}$ \\
6 & $0 \cdot 2998 \times 10^{-1}$ & $0 \cdot 1918 \times 10^{-3}$ \\
7 & $0.5032 \times 10^{-6}$ & $0.9965 \times 10^{-5}$ \\
\hline
\end{tabular}

plotted against the radial displacements under the load. A snap-through mechanism is observed when displacement under the point load reaches a value of 183. The snap-through behaviour can also be observed by comparing equilibrium configurations depicted in Figures 14(c) and 14(d). The response of the shell can be divided into two parts: the first is characterized by bending stiffness and a small slope of the load deflection curve and the second is characterized by the stiffer response of the shell after the snap-through occurs. The first part of the shell response is in accordance with the results in Reference 27, while the snap-through mechanism is not observed by Simo and Kennedy and therefore the second part of the response differs from those reported in Reference 27.

The Euclidean norm of residuals $\|\mathbf{G}\|$ for a typical load step given in Table III again illustrate quadratic rate of asymptotic convergence.

\section{CONCLUSIONS}

In this work, a model for large deformations of thin elasto-plastic shells is described. The main features of the model can be briefly summarised as follows:

(i) Full non-linear shell kinematics is employed accounting for large displacements and finite rotations of the shell. Finite rotation description of the director field is based on the rotation vector formulation.

(ii) Strains are assumed to be small. This makes finite element implementation of a material model particularly simple, since computational models developed for small strain elastoplastic materials can be easily incorporated within the present approach. In addition, all complex aspects of the material identification are immediately applicable to this model.

(iii) The von Mises yield criterion is used in the description of the elasto-plastic material. However, as mentioned above, any other appropriate computational model already available for elasto-plastic materials can easily be incorporated.

(iv) In agreement with (ii) and (iii), numerical integration over the shell thickness is employed. This is considered important if accurate simulation of elasto-plastic shells is required.

(v) Consistent linearization of the shell kinematics and elasto-plastic material model leading to the Newton-Raphson numerical procedure is performed.

(vi) Typical applications of the present model include failures of elasto-plastic thin shells associated with buckling-type instabilities and/or bending-dominated shell problems resulting in formation of plastic hinges. 
A range of selected numerical examples is chosen among the available experimental results and representative numerical tests to illustrate the above points (i)-(vi). Clearly the present approach has shown efficient and robust performance in series of difficult tests.

Future work will be directed towards extension of the present model by incorporating full finite strain kinematics and formulation of the framework for dynamic analysis.

\section{APPENDIX I}

\section{Second directional derivative of the director motion}

In this section, equation (101) is given in the form suitable for the finite element implementation. By employing the following relations (which may be obtained after a straightforward tensor algebra, see e.g. Reference 17):

$$
\begin{aligned}
(\delta \mathbf{s} \cdot \mathbf{s})(\mathbf{s} \cdot \Delta \mathbf{s}) \mathbf{T} & =\left[(\delta \mathbf{s})^{\mathrm{T}}\left[\mathbf{s} \mathbf{s}^{\mathrm{T}}\right] \Delta \mathbf{s}\right] \mathbf{T} \\
(\delta \mathbf{s} \cdot \Delta \mathbf{s}) \mathbf{s} \times \mathbf{T} & =\left[(\delta \mathbf{s})^{\mathrm{T}} \mathbf{I} \Delta \mathbf{s}\right] \mathbf{s} \times \mathbf{T} \\
(\delta \mathbf{s} \cdot \Delta \mathbf{s}) \mathbf{T} & =\left[(\delta \mathbf{s})^{\mathrm{T}} \mathbf{I} \Delta \mathbf{s}\right] \mathbf{T} \\
(\delta \mathbf{s} \cdot \mathbf{s})(\mathbf{s} \cdot \Delta \mathbf{s}) \mathbf{s} \times \mathbf{T} & =\left[(\delta \mathbf{s})^{\mathrm{T}}\left[\mathbf{s s}^{\mathrm{T}}\right] \Delta \mathbf{s}\right] \mathbf{s} \times \mathbf{T} \\
(\mathbf{s} \cdot \Delta \mathbf{s}) \delta \mathbf{s} \times \mathbf{T} & =\left[(\delta \mathbf{s})^{\mathrm{T}} \mathbf{A} \Delta \mathbf{s}\right] \mathbf{E}_{1}+\left[(\delta \mathbf{s})^{\mathrm{T}} \mathbf{B} \Delta \mathbf{s}\right] \mathbf{E}_{2} \\
(\mathbf{s} \cdot \delta \mathbf{s}) \Delta \mathbf{s} \times \mathbf{T} & =\left[(\delta \mathbf{s})^{\mathrm{T}} \mathbf{A}^{\mathrm{T}} \Delta \mathbf{s}\right] \mathbf{E}_{1}+\left[(\delta \mathbf{s})^{\mathrm{T}} \mathbf{B}^{\mathrm{T}} \Delta \mathbf{s}\right] \mathbf{E}_{2}
\end{aligned}
$$

equation (101) expressing the second derivative of the director field motion may be rewritten as

$$
\begin{aligned}
\Delta(\delta \mathbf{t})= & \frac{\|\mathbf{s}\| \cos (\|\mathbf{s}\|)-\sin (\|\mathbf{s}\|)}{\|\mathbf{s}\|^{3}}\left(-\left[(\delta \mathbf{s})^{\mathrm{T}}\left[\mathbf{s} \mathbf{s}^{\mathrm{T}}\right] \Delta \mathbf{s}\right] \mathbf{T}+\left[(\delta \mathbf{s})^{\mathrm{T}} \mathbf{I} \Delta \mathbf{s}\right] \mathbf{s} \times \mathbf{T}\right) \\
& -\frac{\sin (\|\mathbf{s}\|)}{\|\mathbf{s}\|}\left[(\delta \mathbf{s})^{\mathrm{T}} \mathbf{I} \Delta \mathbf{s}\right] \mathbf{T} \\
& +\frac{\sin (\|\mathbf{s}\|)\left(3-\|\mathbf{s}\|^{2}\right)-3\|\mathbf{s}\| \cos (\|\mathbf{s}\|)}{\|\mathbf{s}\|^{5}}\left[(\delta \mathbf{s})^{\mathrm{T}}\left[\mathbf{s} \mathbf{s}^{\mathrm{T}}\right] \Delta \mathbf{s}\right] \mathbf{s} \times \mathbf{T} \\
& +\frac{\|\mathbf{s}\| \cos (\|\mathbf{s}\|)-\sin (\|\mathbf{s}\|)}{\|\mathbf{s}\|^{3}}\left(\mathbf{E}_{1}\left[(\delta \mathbf{s})^{\mathrm{T}}\left[\mathbf{A}+\mathbf{A}^{\mathrm{T}}\right] \Delta \mathbf{s}\right]+\mathbf{E}_{2}\left[(\delta \mathbf{s})^{\mathrm{T}}\left[\mathbf{B}+\mathbf{B}^{\mathrm{T}}\right] \Delta \mathbf{s}\right]\right)
\end{aligned}
$$

Further insight into the constituents to the above equation may be accomplished by resolving vectors $\delta \mathbf{s}, \Delta \mathbf{s}$ and $\mathbf{s}$ in the local orthonormal basis $\left\{\mathbf{E}_{i}\right\}$ (see Section 2.3.2). It then follows that

$$
\delta \mathbf{s}=\delta \hat{s}^{i} \mathbf{E}_{i}=\left\{\begin{array}{l}
\delta \hat{s}^{1} \\
\delta \hat{s}^{2}
\end{array}\right\}, \quad \Delta \mathbf{s}=\Delta \hat{s}^{i} \mathbf{E}_{i}=\left\{\begin{array}{l}
\Delta \hat{S}^{1} \\
\Delta \hat{s}^{2}
\end{array}\right\}, \quad \mathbf{s s}^{\mathrm{T}}=\hat{s}^{i} \hat{S}^{j} \mathbf{E}_{i} \mathbf{E}_{j}^{\mathrm{T}}=\left[\begin{array}{ll}
\hat{S}^{1} \hat{S}^{1} & \hat{S}^{1} \hat{S}^{2} \\
\hat{S}^{1} \hat{S}^{2} & \hat{S}^{2} \hat{S}^{2}
\end{array}\right]
$$

while matrices $\mathbf{A}$ and $\mathbf{B}$ may be expressed as

$$
\mathbf{A}=\left[\begin{array}{ll}
0 & 0 \\
\hat{S}^{1} & \hat{s}^{2}
\end{array}\right], \quad \mathbf{B}=\left[\begin{array}{rr}
-\hat{s}^{1} & -\hat{s}^{2} \\
0 & 0
\end{array}\right]
$$


By multiplying $\Delta(\delta \mathbf{t})$ with any vector $\mathbf{b} \in \mathbb{R}^{3}$ the following expression may be obtained:

$$
\Delta(\delta \mathbf{t}) \cdot \mathbf{b}=\left\{\begin{array}{l}
\delta \hat{s}^{1} \\
\delta \hat{s}^{2}
\end{array}\right\}^{\mathrm{T}} \mathbf{Y}\left\{\begin{array}{l}
\Delta \hat{s}^{1} \\
\Delta \hat{s}^{2}
\end{array}\right\}
$$

where the matrix $\mathbf{Y}$ is given as

$$
\begin{aligned}
\mathbf{Y}= & \frac{\|\mathbf{s}\| \cos (\|\mathbf{s}\|)-\sin (\|\mathbf{s}\|)}{\|\mathbf{s}\|^{3}}\left(-\left[\begin{array}{cc}
\hat{S}^{1} \hat{S}^{1} & \hat{S}^{1} \hat{S}^{2} \\
\hat{S}^{1} \hat{S}^{2} & \hat{S}^{2} \hat{S}^{2}
\end{array}\right] \mathbf{T} \cdot \mathbf{b}+\left[\begin{array}{ll}
1 & 0 \\
0 & 1
\end{array}\right](\mathbf{s} \times \mathbf{T}) \cdot \mathbf{b}\right) \\
& -\frac{\sin (\|\mathbf{s}\|)}{\|\mathbf{s}\|}\left[\begin{array}{cc}
1 & 0 \\
0 & 1
\end{array}\right] \mathbf{T} \cdot \mathbf{b} \\
& +\frac{\sin (\|\mathbf{s}\|)\left(3-\|\mathbf{s}\|^{2}\right)-3\|\mathbf{s}\| \cos (\|\mathbf{s}\|)}{\|\mathbf{s}\|^{5}}\left[\begin{array}{ll}
\hat{S}^{1} \hat{S}^{1} & \hat{S}^{1} \hat{S}^{2} \\
\hat{S}^{1} \hat{S}^{2} & \hat{S}^{2} \hat{S}^{2}
\end{array}\right](\mathbf{s} \times \mathbf{T}) \cdot \mathbf{b} \\
& +\frac{\|\mathbf{s}\| \cos (\|\mathbf{s}\|)-\sin (\|\mathbf{s}\|)}{\|\mathbf{s}\|^{3}}\left(\left[\begin{array}{cc}
0 & \hat{S}^{1} \\
\hat{S}^{1} & 2 \hat{S}^{2}
\end{array}\right] \mathbf{E}_{1} \cdot \mathbf{b}-\left[\begin{array}{cc}
2 \hat{S}^{1} & \hat{S}^{2} \\
\hat{S}^{2} & 0
\end{array}\right] \mathbf{E}_{2} \cdot \mathbf{b}\right)
\end{aligned}
$$

Note that the vector $\mathbf{s}$, which can be resolved in the fixed basis $\left\{\mathbf{e}_{1}, \mathbf{e}_{2}, \mathbf{e}_{3}\right\}$ as $\mathbf{s}=s^{i} \mathbf{e}_{i}$, may be alternatively obtained as (see (22) and (23))

$$
\left\{\begin{array}{c}
s^{1} \\
s^{2} \\
s^{3}
\end{array}\right\}=\left[\begin{array}{cc}
\Lambda_{11}^{0} & \Lambda_{12}^{0} \\
\Lambda_{21}^{0} & \Lambda_{22}^{0} \\
\Lambda_{31}^{0} & \Lambda_{32}^{0}
\end{array}\right]\left\{\begin{array}{c}
\hat{s}^{1} \\
\hat{s}^{2}
\end{array}\right\}=\left[\mathbf{E}_{1}, \mathbf{E}_{2}\right]\left\{\begin{array}{c}
\hat{s}^{1} \\
\hat{s}^{2}
\end{array}\right\}
$$

\section{REFERENCES}

1. J. Lemaitre and J.-L. Chaboche, Mechanics of Solid Materials, Cambridge University Press, Cambridge, 1990.

2. J. C. Simo and R. L. Taylor, 'A return mapping algorithm for plane stress elastoplasticity', Int. j. numer. methods eng., 22, 649-670 (1986).

3. E. Ramm and A. Matzenmiller, 'Computational aspects of elasto-plasticity in shell analysis', in D. R. J. Owen et al. (eds), Computational Plasticity I: Models, Software and Applications, Pineridge Press, Swansea, 1987, pp. 711-734.

4. J. C. Simo and D. D. Fox, 'On a stress resultant geometrically exact shell model. Part III: computational aspects of the nonlinear theory', Comput. Methods Appl. Mech. Eng., 79, 21-70 (1990).

5. H. Parisch, 'An investigation of a finite rotation four node assumed strain shell element', Int. j. numer. methods eng., $\mathbf{3 1}$, 127-150 (1991)

6. N. Stander, A. Matzenmiller and E. Ramm, 'An assessment of assumed strain methods in finite rotation shell analysis', Eng. Comput., 6, 57-66 (1989).

7. C. Sansour and H. Bufler, 'An exact finite rotation shell theory, its mixed variational formulation and its finite element implementation', Int. j. numer. methods eng., 34, 73-115 (1992).

8. P. Wriggers and F. Gruttmann, 'Thin shells with finite rotations formulated in Biot stresses: Theory and finite element formulation', Int. j. numer. methods eng., 36, 2049-2071 (1993).

9. N. Büchter and E. Ramm, 'Shell theory versus degeneration - a comparison in large rotation finite element analysis', Int. j. numer. methods eng., 34, 39-59 (1992).

10. P. M. Naghdi, 'The theory of shells', in S. Flüge (ed.), Handbuch der Physik VI/2, Springer, Berlin, 1972.

11. J. C. Simo and D. D. Fox, 'On a stress resultant geometrically exact shell model. Part I: formulation and optimal parametrization', Comput. Methods Appl. Mech. Eng., 72, 267-304 (1987).

12. J. C. Simo, M. S. Rifai and D. D. Fox, 'On a stress resultant geometrically exact shell model. Part IV: Variable thickness shells with through-the-thickness stretching', Comput. Methods Appl. Mech. Eng., 81, 91-126 (1990).

13. B. Brank, D. Perić and F. B. Damjanić, 'On implementation of a nonlinear four-node finite element for thin multilayered elastic shells', Comput. Mech., 16, 341-359 (1995).

14. E. N. Dvorkin and K. J. Bathe, 'A continuum mechanics based four-node shell element for general nonlinear analysis', Eng. Comput., 1, 77-88 (1984).

15. G. Jelenić, 'Large deformations of elastic beams in 3-D space' (in Slovenian), Ph.D. Thesis, University of Ljubljana, 1993.

16. W. Pietraszkiewicz and J. Badur, 'Finite rotations in the description of continuum deformation', Int. J. Eng. Sci., 21, 1097-1115 (1983). 
17. M. E. Gurtin, An Introduction to Continuum Mechanics, Academic Press, Orlando, 1981.

18. N. Büchter and E. Ramm, '3D-extension of nonlinear shell equations based on the enhanced assumed strain concept', in Ch. Hirsch, J. Périaux and E. Oñate (eds), Computational Methods in Applied Sciences, Invited Lectures on Special Technologies Sessions of the 1st European CFD Conference and the First European Conference on Numerical Methods in Engineering, Brussels, Belgium, 7-11 September 1992, Elsevier, Amsterdam, 1992, pp. 55-62.

19. J. C. Simo, 'On a stress resultant geometrically exact shell model. Part VII: Shell intersections with 5/6-DOF finite element formulations', Comput. Methods Appl. Mech. Eng., 108, 319-339 (1993).

20. F. I. Niordson, Shell Theory, North-Holland, Amsterdam, 1985.

21. E. H. Lee, 'Elastic-plastic deformations at finite strains', J. Appl. Mech., 36, 1-6 (1969).

22. M. Ortiz and E. P. Popov, 'Accuracy and stability of integration algorithms for elastoplastic constitutive relations', Int. j. numer. methods eng., 21, 1561-1576 (1985).

23. M. A. Crisfield, Non-linear Finite Element Analysis of Solids and Structures, Vol. 1, Wiley, Chichester, 1991.

24. D. Perić and D. R. J. Owen, 'The Morley thin shell finite element for large deformations problems: simplicity versus sophistication', in N. Bićanić (ed.), Proc. Int. Conf. on Nonlinear Engineering Computations, Pineridge Press, Swansea, 1991, pp. 121-142.

25. M. Crisfield and X. Peng, 'Efficient non-linear shell formulations with large rotations and plasticity', in D. R. J. Owen et al. (eds), Computational Plasticity III: Fundamentals and Applications, Pineridge Press, Swansea, 1992, pp. 1979-1996.

26. L. H. Sobel and S. Z. Newman, 'Plastic buckling of cylindrical shells under axial compression', J. Press. Vess. Technol., 102, 40-44 (1980).

27. J. C. Simo and J. G. Kennedy, 'On a stress resultant geometrically exact shell model. Part V: Nonlinear plasticity: formulation and integration algorithms', Comput. Methods Appl. Mech. Eng., 96, 133-171 (1992). 